\title{
Asymptotic ideals (ideals in the ring of Colombeau generalized constants with continuous parametrization)
}

\author{
A. Khelif, D. Scarpalezos ${ }^{\dagger}$ H. Vernaeve ${ }^{\ddagger}$
}

\begin{abstract}
We study the asymptotics at zero of continuous functions on $(0,1]$ by means of their asymptotic ideals, i.e., ideals in the ring of continuous functions on $(0,1]$ satisfying a polynomial growth condition at 0 modulo rapidly decreasing functions at 0 . As our main result, we characterize maximal and prime ideals in terms of maximal and prime filters.
\end{abstract}

\section{Introduction}

In this paper, we study the asymptotic ideals of continuous functions $(0,1] \rightarrow \mathbb{K}$ (where $\mathbb{K}$ is one of the fields $\mathbb{R}$ or $\mathbb{C}$ ), i.e., ideals in the ring of continuous functions $\phi$ satisfying the following growth condition (usually called moderateness)

$$
(\exists N \in \mathbb{N})\left(\exists \varepsilon_{0}>0\right)\left(\forall \varepsilon \leq \varepsilon_{0}\right)|\phi(\varepsilon)| \leq \varepsilon^{-N}
$$

modulo the ideal of continuous functions $\phi$ satisfying

$$
(\forall n \in \mathbb{N})\left(\exists \varepsilon_{0}>0\right)\left(\forall \varepsilon \leq \varepsilon_{0}\right)|\phi(\varepsilon)| \leq \varepsilon^{n}
$$

(usually called negligibility). Apart from the obvious interest of such a study to asymptotic analysis, such equivalence classes of functions also naturally arise in generalized function theory as the ring of generalized constants $\widetilde{\mathbb{K}}_{\text {cnt }}$ of the algebra of Colombeau generalized functions (see \$2).

The ring $\widetilde{\mathbb{K}}_{\text {cnt }}$ of generalized constants with continuous dependence on the parameter has been introduced and studied in [5], where it is also shown that this ring is isomorphic to the ring of generalized constants with smooth dependence. In fact, the study of the ring $\widetilde{\mathbb{K}}_{\text {cnt }}$ amounts to the study of the asymptotics at zero of moderate continuous functions on $(0,1]$.

In generalized function theory, the choice of continuous dependence comes from the observation that when one embeds distributions in an algebra of Colombeau generalized

\footnotetext{
*Université Paris 7, Equipe de Logique

†Université Paris 7, Centre de Mathématiques

$\ddagger$ Ghent University, Department of Mathematics
} 
functions and when one solves nonlinear problems, one always encounters generalized functions represented by continuous (even smooth) nets of smooth functions.

The algebraic properties of the ring $\widetilde{\mathbb{K}}_{\text {cnt }}$ are different from those of the ring $\widetilde{\mathbb{K}}$ of generalized constants without continuous dependence on the parameter, and many tools used in the study of $\widetilde{\mathbb{K}}$ cannot be used. Most strikingly, this is manifested by the fact that $\widetilde{\mathbb{K}}_{\mathrm{cnt}}$ does not have any nontrivial idempotent elements, in sharp contrast with the ring $\widetilde{\mathbb{K}}$ (which is a so-called exchange ring [13]). Thus the main tools used in [1] and [13] to study $\widetilde{\mathbb{K}}$ cannot be used.

In this paper, we study prime and maximal ideals by attaching a filter of closed subsets of $(0,1]$ to each ideal. The filter is analogous to the filter $\left\{S \subseteq(0,1]: e_{S^{c}} \in I\right\}$ attached to an ideal $I \triangleleft \widetilde{\mathbb{K}}([13, \S 6])$, and thus allows us to overcome the difficulty of the lack of idempotents. In this way, we obtain a classification of maximal and minimal prime ideals in terms of maximal and prime filters.

The methods used in this paper are inspired by the study of the ideals in $\widetilde{\mathbb{K}}[1,13$, and by the study of maximal ideals of rings of continuous functions by Gillman and Jerison [7]. Compared to [7], the main novelty is the adaptation to the asymptotic nature of the ring $\widetilde{\mathbb{K}}_{\text {cnt }}$.

\section{Preliminaries}

The ring $\widetilde{\mathbb{K}}$, with $\mathbb{K}=\mathbb{R}$ or $\mathbb{K}=\mathbb{C}$ (the field of real, resp. complex numbers), is defined as $\mathcal{M}_{\mathbb{K}} / \mathcal{N}_{\mathbb{K}}$, where

$$
\begin{aligned}
\mathcal{M}_{\mathbb{K}} & =\left\{\left(x_{\varepsilon}\right)_{\varepsilon} \in \mathbb{K}^{(0,1]}:(\exists N \in \mathbb{N})\left(\exists \varepsilon_{0}>0\right)\left(\forall \varepsilon \leq \varepsilon_{0}\right)\left|x_{\varepsilon}\right| \leq \varepsilon^{-N}\right\} \\
\mathcal{N}_{\mathbb{K}} & =\left\{\left(x_{\varepsilon}\right)_{\varepsilon} \in \mathbb{K}^{(0,1]}:(\forall n \in \mathbb{N})\left(\exists \varepsilon_{0}>0\right)\left(\forall \varepsilon \leq \varepsilon_{0}\right)\left|x_{\varepsilon}\right| \leq \varepsilon^{n}\right\} .
\end{aligned}
$$

We denote by $\left[x_{\varepsilon}\right] \in \widetilde{\mathbb{K}}$ the element with representative $\left(x_{\varepsilon}\right)_{\varepsilon}$ and we denote $\rho:=[\varepsilon]$. $\widetilde{\mathbb{K}}$ is a complete topological ring with the so-called sharp topology, which can be defined as follows. Let $x=\left[x_{\varepsilon}\right] \in \widetilde{\mathbb{K}}$. Let

$$
v(x):=\sup \left\{a \in \mathbb{R}:\left(\exists \varepsilon_{0}>0\right)\left(\forall \varepsilon \leq \varepsilon_{0}\right)\left|x_{\varepsilon}\right| \leq \varepsilon^{a}\right\} .
$$

Then the ultrametric $d(x, y):=e^{-v(x-y)}$ induces a topology on $\widetilde{\mathbb{K}}$ which is called the sharp topology [12].

Denoting by $\mathcal{C}((0,1])$ (resp. $\mathcal{C}^{\infty}((0,1])$ the set of continuous (resp. smooth) maps in $\mathbb{K}^{(0,1]}$, the ring $\widetilde{\mathbb{K}}_{\mathrm{cnt}}:=\left(\mathcal{M}_{\mathbb{K}} \cap \mathcal{C}((0,1])\right) /\left(\mathcal{N}_{K} \cap \mathcal{C}((0,1])\right)$ and $\widetilde{\mathbb{K}}_{\mathrm{sm}}:=\left(\mathcal{M}_{\mathbb{K}} \cap\right.$ $\left.\mathcal{C}^{\infty}((0,1])\right) /\left(\mathcal{N}_{K} \cap \mathcal{C}^{\infty}((0,1])\right)$. Clearly, $\widetilde{\mathbb{K}}_{\mathrm{sm}} \subseteq \widetilde{\mathbb{K}}_{\mathrm{cnt}} \subseteq \widetilde{\mathbb{K}}$. In [5], it is shown that $\widetilde{\mathbb{K}}_{\text {cnt }}=\widetilde{\mathbb{K}}_{\mathrm{sm}}$.

We denote $I \triangleleft \widetilde{\mathbb{K}}_{\text {cnt }}$ for a proper ideal $I$ of $\widetilde{\mathbb{K}}_{\text {cnt }}$ (i.e., $I \neq \widetilde{\mathbb{K}}_{\text {cnt }}$ ).

$\widetilde{\mathbb{K}}$ is an exchange ring [13], i.e., for each $a \in \widetilde{\mathbb{K}}$, there exists an idempotent $e \in \widetilde{\mathbb{K}}$ such that $a+e$ is invertible. Unlike $\widetilde{\mathbb{K}}, \widetilde{\mathbb{K}}_{\text {cnt }}$ is not an exchange ring [5, Lemma 4.3].

Like $\widetilde{\mathbb{K}}, \widetilde{\mathbb{K}}_{\text {cnt }}$ is a Gelfand ring [5, Lemma 4.5], i.e., every prime ideal is contained in a unique maximal ideal.

Like $\widetilde{\mathbb{K}}, \widetilde{\mathbb{K}}_{\mathrm{cnt}}$ is a Bezout ring [5, Prop. 4.26], i.e., every finitely generated ideal is principal.

Like $\widetilde{\mathbb{R}}, \widetilde{\mathbb{R}}_{\text {cnt }}$ is an l-ring (or lattice-ordered ring) [5, Prop. 4.13]. 
Let $I \unlhd \widetilde{\mathbb{K}}_{\text {cnt }}$ and $x \in I$. Then $|x| \in I$ [5, Lemma 4.24].

Let $I \unlhd \widetilde{\mathbb{R}}_{\text {cnt }}$. Then $I$ is an l-ideal (or absolutely (order) convex), i.e., if $x \in I, x^{\prime} \in \widetilde{\mathbb{R}}_{\text {cnt }}$ and $\left|x^{\prime}\right| \leq|x|$, then $x^{\prime} \in I$. [5, Prop. 4.25].

Let us point out explicitly the corollary that then also for $I \unlhd \widetilde{\mathbb{C}}_{\mathrm{cnt}}, z \in I, z^{\prime} \in \widetilde{\mathbb{C}}_{\mathrm{cnt}}$, $\left|z^{\prime}\right| \leq|z|$ implies that $z^{\prime} \in I$. Indeed, $z \in I$ implies $|z| \in I \cap \widetilde{\mathbb{R}}_{\text {cnt }}$ [5, Lemma 4.24]. As $I \cap \widetilde{\mathbb{R}}_{\mathrm{cnt}} \unlhd \widetilde{\mathbb{R}}_{\mathrm{cnt}}, I \cap \widetilde{\mathbb{R}}_{\mathrm{cnt}}$ is an l-ideal in $\widetilde{\mathbb{R}}_{\mathrm{cnt}}$. Hence $\left|\Re z^{\prime}\right| \leq|z|$ implies that $\Re z^{\prime} \in I \cap \widetilde{\mathbb{R}}_{\mathrm{cnt}}$. Similarly, $\Im z^{\prime} \in I \cap \widetilde{\mathbb{R}}_{\text {cnt }}$. Thus $z^{\prime}=\Re z^{\prime}+i \Im z^{\prime} \in I$.

Hence the bijective correspondence of ideals in $\widetilde{\mathbb{K}}_{\text {cnt }}$ takes the same form as for ideals in $\widetilde{\mathbb{K}}([13])$ : the map $I \unlhd \widetilde{\mathbb{C}}_{\text {cnt }} \mapsto I \cap \widetilde{\mathbb{R}}_{\text {cnt }}=\{\Re z: z \in I\} \unlhd \widetilde{\mathbb{R}}_{\text {cnt }}$ has as an inverse the map $J \unlhd \widetilde{\mathbb{R}}_{\text {cnt }} \mapsto\langle J\rangle=\left\{z \in \widetilde{\mathbb{C}}_{\text {cnt }}:|z| \in J\right\} \unlhd \widetilde{\mathbb{C}}_{\text {cnt }}$ (where $\langle J\rangle$ is also the ideal generated by $J$ in $\left.\widetilde{\mathbb{C}}_{\text {cnt }}\right)$. It is an inclusion-preserving bijection between the lattice of ideals of $\widetilde{\mathbb{C}}_{\text {cnt }}$ and the lattice of ideals of $\widetilde{\mathbb{R}}_{\text {cnt }}$. In particular, arbitrary sums and intersections are preserved. One easily checks that the isomorphism also preserves products of ideals, principal, pseudoprime and irreducible ideals.

Let $R$ be a commutative ring with 1 . An ideal $I \unlhd R$ is pure if [4, Prop. 7.2]

$$
(\forall x \in I)(\exists y \in I)(x=x y) .
$$

We denote by $m(I)$ the pure part of $I \unlhd R$, i.e., the largest pure ideal contained in $I$ [4, Prop. 7.8]. By definition, $I$ is pure iff $I=m(I)$. If $R$ is a Gelfand ring, then [4, $\S 8.2-3]$

$$
m(I)=\{x \in R:(\exists y \in I)(x=x y)\} .
$$

An ideal $I \unlhd R$ is idempotent if $I^{2}=I$.

We denote the radical of $I \unlhd R$ by $\sqrt{I}=\left\{x \in R:(\exists n \in \mathbb{N}) x^{n} \in I\right\}=\bigcap_{P \text { prime }}^{I \subseteq P} P$ (e.g., see [7, 0.18]).

$I \unlhd R$ is radical (or semiprime) if $I=\sqrt{I}$, or equivalently, if $(\forall x \in R)\left(x^{2} \in I \Rightarrow x \in I\right)$. $I \unlhd R$ is pseudoprime if for each $a, b \in R, a b=0$ implies $a \in I$ or $b \in I$.

$I \unlhd R$ is irreducible (or meet-irreducible) if for each $J, K \unlhd R, I=J \cap K$ implies $I=J$ or $I=K[10, \S 6]$.

\section{Characteristic sets}

Definition 3.1. A set $S \subseteq(0,1]$ such that $0 \in \bar{S}$ (closure in $\mathbb{R}$ ) is called a characteristic set [5]. We denote the set of all characteristic sets by $\mathcal{S}$.

Let $S, T \in \mathcal{S}$. We say that $T$ is an extension of $S$ if $\bar{S} \subseteq T^{\circ}$ (closure and interior in $(0,1]$ ) and denote this by $S \prec T$ (or equivalently, $T \succ S$ ). It is straightforward to check that $\prec$ is antireflexive and transitive on $\mathcal{S} \backslash\{(0,1]\}$, and hence defines a partial order on $\mathcal{S} \backslash\{(0,1]\}$. Notice that $(0,1] \prec(0,1]$, which will turn out to be convenient.

Lemma 3.2. Let $S, T \in \mathcal{S}$.

1. If $S \prec T$, there exists $U \in \mathcal{S}$ such that $S \prec U \prec T$. In particular, $\prec$ is a dense order on $\mathcal{S} \backslash\{(0,1]\}$.

2. $S \prec T$ iff $T^{c} \prec S^{c}$. 
Proof. 1. Let $S \prec T$. By Urysohn's lemma, there exists $\phi \in \mathcal{C}((0,1])$ such that $0 \leq \phi \leq 1, \phi_{\mid S}=0$ and $\phi_{\mid T^{c}}=1$. Let $U:=\{\varepsilon \in(0,1]: \phi(\varepsilon) \leq 1 / 2\}$. Then $S \prec U \prec T$.

2. $\bar{S} \subseteq T^{\circ} \Longleftrightarrow \overline{\left(T^{c}\right)}=\left(T^{\circ}\right)^{c} \subseteq(\bar{S})^{c}=\left(S^{c}\right)^{\circ}$.

Definition 3.3. (cf. [5, 4.16]) Let $x \in \widetilde{\mathbb{K}}_{\mathrm{cnt}}$ and $S \in \mathcal{S}$. Then $x_{\mid S}=0$ if

$$
(\forall n \in \mathbb{N})(\exists \delta>0)(\forall \varepsilon \in S \cap(0, \delta))\left(\left|x_{\varepsilon}\right| \leq \varepsilon^{n}\right) .
$$

where $\left(x_{\varepsilon}\right)_{\varepsilon}$ is any representative of $x$. We similarly write $x_{\mid S}=y_{\mid S}$ for $(x-y)_{\mid S}=0$, $x_{\mid S}=1$ for $(x-1)_{\mid S}=0, \ldots$

We say that $x_{\mid S}$ is invertible if there exists $y \in \widetilde{\mathbb{K}}_{\mathrm{cnt}}$ such that $(x y)_{\mid S}=1$.

Lemma 3.4. Let $S \in \mathcal{S}$.

1. Let $x \in \widetilde{\mathbb{K}}_{\mathrm{cnt}}$. Then the following are equivalent:

(a) $x_{\mid S}$ is invertible (in $\widetilde{\mathbb{K}}_{\text {cnt }}$ )

(b) $x_{\mid S}$ is invertible in $\widetilde{\mathbb{K}}$

(c) $x_{\mid S}$ is bounded away from zero, i.e., for some representative $\left(x_{\varepsilon}\right)_{\varepsilon}$ of $x$,

$$
(\exists n \in \mathbb{N})(\exists \delta>0)(\forall \varepsilon \in S \cap(0, \delta))\left(\left|x_{\varepsilon}\right| \geq \varepsilon^{n}\right) .
$$

(the statement then automatically holds for any representative $\left(x_{\varepsilon}\right)_{\varepsilon}$ of $x$ ).

(d) for each characteristic set $T \subseteq S, x_{\mid T} \neq 0$.

2. $\left\{x \in \widetilde{\mathbb{K}}_{\mathrm{cnt}}: x_{\mid S}\right.$ is invertible $\}$ is open.

3. $x_{\mid S}=0$ iff for each characteristic set $T \subseteq S, x_{\mid T}$ is not invertible.

Proof. 1. $(b) \Leftrightarrow(c) \Leftrightarrow(d)$ : by [13, Lemma 4.1].

$(a) \Rightarrow(b)$ : trivial.

$(c) \Rightarrow(a)$ : let $T:=\left\{\varepsilon \in(0,1]:\left|x_{\varepsilon}\right|>\varepsilon^{n} / 2\right\}$. As $\left(x_{\varepsilon}\right)_{\varepsilon}$ is continuous, $S \cap(0, \delta) \prec T$.

By Urysohn's lemma, there exists $\phi \in \mathcal{C}((0,1])$ such that $0 \leq \phi \leq 1, \phi_{\mid S \cap(0, \delta)}=1$ and $\phi_{\mid T^{c}}=0$. Let $y_{\varepsilon}:=\phi(\varepsilon) / x_{\varepsilon}$, if $\varepsilon \in T$ and $y_{\varepsilon}:=0$, if $\varepsilon \in T^{c}$. Then $\left|y_{\varepsilon}\right| \leq 2 \varepsilon^{-n},\left(y_{\varepsilon}\right)_{\varepsilon} \in$ is continuous and $x_{\varepsilon} y_{\varepsilon}=1$ for each $\varepsilon \in S \cap(0, \delta)$. Hence $\left(y_{\varepsilon}\right)_{\varepsilon}$ is a representative of some $y \in \widetilde{\mathbb{K}}_{\mathrm{cnt}}$ with $(x y)_{\mid S}=1$.

2. Let $x_{\mid S}$ be invertible. Let $n \in \mathbb{N}$ as in part $1(\mathrm{c})$. Then $y_{\mid S}$ is invertible for each $y \in \widetilde{\mathbb{K}}_{\text {cnt }}$ with $|x-y| \leq \rho^{n} / 2$ (again by part $1(\mathrm{c})$ ).

3. By [13, Lemma 4.1], since $\widetilde{\mathbb{K}}_{\text {cnt }} \subseteq \widetilde{\mathbb{K}}$.

Proposition 3.5. Let $x \in \widetilde{\mathbb{K}}_{\mathrm{cnt}}$ and $S \in \mathcal{S}$.

1. If $x_{\mid S}=0$, then $x_{\mid T}=0$ for some $T \succ S$.

2. If $x_{\mid S}$ is invertible, then $x_{\mid T}$ is invertible for some $T \succ S$. 
Proof. 1. Let $\left(x_{\varepsilon}\right)_{\varepsilon \in(0,1]}$ be a (continuous) representative of $x$. Then for each $n \in \mathbb{N}$, there exist $\delta_{n}>0$ (w.l.o.g. strictly decreasing and tending to 0 ) such that $\left|x_{\varepsilon}\right| \leq \varepsilon^{n}$ for each $\varepsilon \in S, \varepsilon \leq \delta_{n}$. Then let $T:=\bigcup_{n \in \mathbb{N}}\left(\delta_{n+2}, \delta_{n}\right) \cap\left\{\varepsilon \in(0,1]:\left|x_{\varepsilon}\right| \leq 2 \varepsilon^{n}\right\}$. Then also $x_{\mid T}=0$. We show that $S \prec T$. Let $\varepsilon \in \bar{S}$. Then $\varepsilon \in\left(\delta_{n+2}, \delta_{n}\right)$ for some $n$. By continuity, also $\left|x_{\varepsilon}\right| \leq \varepsilon^{n}$ for each $\varepsilon \in \bar{S}, \varepsilon<\delta_{n}$. Hence $\varepsilon$ belongs to the open set $\left(\delta_{n+2}, \delta_{n}\right) \cap\left\{\varepsilon \in(0,1]:\left|x_{\varepsilon}\right|<2 \varepsilon^{n}\right\} \subseteq T$. Thus $\varepsilon \in T^{\circ}$.

2. Let $n \in \mathbb{N}$ and $\delta>0$ as in lemma 3.4.1(c). Let $T:=\left\{\varepsilon \in(0,1]:\left|x_{\varepsilon}\right|>\varepsilon^{n} / 2\right\} \cup$ $(\delta / 2,1)$. As $\left(x_{\varepsilon}\right)_{\varepsilon}$ is continuous, $S \prec T$. By lemma 3.4.1(c), $x_{\mid T}$ is invertible.

Lemma 3.6. Let $a, b \in \widetilde{\mathbb{K}}_{\text {cnt }}$ and $S \in \mathcal{S}$. If $(a b)_{\mid S}=0$, then there exist closed $T, U$ with $S \subseteq T^{\circ} \cup U^{\circ}$ such that $a_{\mid T}=0$ and $b_{\mid U}=0$.

Proof. As $a, b \in \widetilde{\mathbb{K}}$, there exists $V \subseteq S$ such that $a_{\mid V}=0$ and $b_{\mid S \backslash V}=0$ [13]. As $a, b \in \widetilde{\mathbb{K}}_{\text {cnt }}$, there exist (w.l.o.g. closed) $T, U$ with $V \prec T, S \backslash V \prec U$ such that $a_{\mid T}=0$ and $b_{\mid U}=0$ by Prop. 3.5.

\section{Asymptotic filters}

In [7], to any ideal $I \triangleleft \mathcal{C}(X)$ (with $X$ a topological space), a filter is associated consisting of the zero-sets of all $f \in I$ and conversely, to a filter $\mathcal{F}$ of zero-sets, an ideal $I$ is associated. Taking into account that there is no largest zero-set for $x \in \widetilde{\mathbb{K}}_{\mathrm{cnt}}$, we proceed as follows:

Definition 4.1. A filter of closed subsets of $(0,1]$ is a family $\mathcal{F}$ of (relatively) closed subsets of $(0,1]$ such that

1. $\emptyset \notin \mathcal{F}$

2. if $S, T \in \mathcal{F}$, then $S \cap T \in \mathcal{F}$

3. if $S \in \mathcal{F}, T \subseteq(0,1]$ is closed and $S \subseteq T$, then $T \in \mathcal{F}$.

A closed characteristic subset of $(0,1]$ is called an asymptotic subset. We denote the set of all asymptotic subsets by $\mathcal{A}$.

An asymptotic filter or a-filter is a filter of closed subsets of $(0,1]$ that contains $(0, \delta]$ for each $\delta>0$. Notice that this implies that $\mathcal{F} \subseteq \mathcal{A}$.

$W e$ define as follows a topology on $\mathcal{A}$. Denoting open intervals corresponding to $\prec$ by

$$
(S, T)_{\prec}:=\{U \in \mathcal{A}: S \prec U \prec T\},
$$

the extension topology is the topology on $\mathcal{A}$ with base $\left\{(S, T)_{\prec}: S, T \subseteq(0,1]\right\}$. We will call $\prec$-open, $\prec$-closed, ... sets that are open, closed, ... for this topology. Notice that $\{(0,1]\}$ is $\prec$-open, which will turn out to be convenient.

Remark 4.2. A filter is called free (or non-principal) if $\bigcap_{S \in \mathcal{F}} S=\emptyset$. We can alternatively define an a-filter as a free filter of closed subsets of $(0,1]$. For, if $\mathcal{F}$ is a filter of closed subsets of $(0,1]$ and $(0, \delta] \notin \mathcal{F}$ for some $\delta>0$, then $S \cap[\delta, 1] \neq \emptyset$ for each $S \in \mathcal{F}$. By compactness of $[\delta, 1]$, it would then follow that $\bigcap_{S \in \mathcal{F}} S \cap[\delta, 1] \neq \emptyset$. 
Definition 4.3. Let $I \triangleleft \widetilde{\mathbb{K}}_{\mathrm{cnt}}$. Then

$$
\mathcal{F}(I):=\left\{S \subseteq(0,1] \text { closed }:(\exists x \in I)\left(x_{\mid S^{c}} \text { is invertible }\right)\right\}
$$

(here it is understood that $x_{\mid S}$ is trivially invertible if $\left.0 \notin \bar{S}\right)$.

Let $\mathcal{F}$ be an a-filter on $(0,1]$. Then

$$
I(\mathcal{F}):=\left\{x \in \widetilde{\mathbb{K}}_{\mathrm{cnt}}:(\exists S \in \mathcal{F})\left(x_{\mid S}=0\right)\right\} .
$$

Lemma 4.4. For $I \triangleleft \widetilde{\mathbb{K}}_{\mathrm{cnt}}$,

$$
\mathcal{F}(I)=\left\{S \subseteq(0,1] \text { closed }:(\exists x \in I)\left(x_{\mid S^{c}}=1\right)\right\} .
$$

Proof. If $x \in I$ and $x_{\mid S^{c}}$ is invertible, then there exists $y \in \widetilde{\mathbb{K}}_{\mathrm{cnt}}$ such that $(x y)_{\mid S^{c}}=1$, and $x y \in I$.

Proposition 4.5. Let $I \triangleleft \widetilde{\mathbb{K}}_{\mathrm{cnt}}$ and $\mathcal{F}$ an a-filter on $(0,1]$.

1. $\mathcal{F}(I)$ is an a-filter on $(0,1]$.

2. $I(\mathcal{F}) \triangleleft \widetilde{\mathbb{K}}_{\mathrm{cnt}}$.

3. $\mathcal{F}(I(\mathcal{F})) \subseteq \mathcal{F}$.

4. $I(\mathcal{F}(I)) \subseteq I$.

Proof. 1. Since a proper ideal does not contain invertible elements, $\emptyset \notin \mathcal{F}(I)$.

If $S, T \in \mathcal{F}(I)$, then there exist $x, y \in I$ such that $x_{\mid S^{c}}$ and $y_{\mid T^{c}}$ are invertible. Hence also $|x|^{2}+|y|^{2} \in I$ and $\left(|x|^{2}+|y|^{2}\right)_{\mid S^{c} \cup T^{c}}$ is invertible, so also $S \cap T \in \mathcal{F}(I)$.

If $S \in \mathcal{F}(I), T \subseteq(0,1]$ is closed and $S \subseteq T$, then clearly $T \in \mathcal{F}(I)$.

If $\delta>0$, then $0 \notin \overline{(0, \delta]^{c}}$, hence $x_{\mid(0, \delta]^{c}}$ is (trivially) invertible for each $x \in \widetilde{\mathbb{K}}_{\mathrm{cnt}}$.

2. If $x, y \in I(\mathcal{F})$, then $x_{\mid S}=0$ and $y_{\mid T}=0$ for some $S, T \in \mathcal{F}$. Then also $x+y_{\mid S \cap T}=0$ and $S \cap T \in \mathcal{F}$, so $x+y \in I(\mathcal{F})$. For $z \in \widetilde{\mathbb{K}}_{\text {cnt }}$, also $x z_{\mid S}=0$, so $x z \in I(\mathcal{F}) .1 \notin I(\mathcal{F})$, since $1_{\mid S} \neq 0$ for each $S \in \mathcal{S}$.

3. Let $S \in \mathcal{F}(I(\mathcal{F}))$. Then there exists $x \in I(\mathcal{F})$ such that $x_{\mid S^{c}}=1$. So there exists $T \in \mathcal{F}$ such that $x_{\mid T}=0$. Then $T \cap(0, \delta] \subseteq S$ for some $\delta>0$. For otherwise, one constructs $V \subseteq T \cap S^{c}$ with $0 \in \bar{V}$ such that $x_{\mid V}=0$, contradicting $x_{\mid V}=1$. Thus $S \in \mathcal{F}$.

4. Let $x \in I(\mathcal{F}(I))$. Then there exists $S \in \mathcal{F}(I)$ such that $x_{\mid S}=0$. So there exists $y \in I$ such that $y_{\mid S^{c}}=1$. As $x \in \widetilde{\mathbb{K}}_{\mathrm{cnt}},|x| \leq \rho^{-N}$ for some $N \in \mathbb{N}$. Then $|x| \leq \rho^{-N}|y|$. As $\rho^{-N} y \in I$ and ideals in $\widetilde{\mathbb{K}}_{\text {cnt }}$ are absolutely order convex [5, Prop. 4.25], $x \in I$.

Proposition 4.6. Let $\mathcal{F}$ be an a-filter on $(0,1]$. Then
1. $\mathcal{F}^{\circ}=\{S \in \mathcal{A}:(\exists T \prec S)(T \in \mathcal{F})\}$ ( $\mathcal{F}^{\circ}$ denotes the $\prec$-interior $)$.
2. $\mathcal{F}^{\circ}$ is an a-filter. 
Proof. 1. $\subseteq$ : let $\mathcal{X} \subseteq \mathcal{F}$ be $\prec$-open. If $S \in \mathcal{X}$, then $S \in(T, U)_{\prec} \subseteq \mathcal{X}$, for some $T, U \subseteq(0,1]$. W.l.og., $T$ is closed. We first show that there exists $V \prec S$ with $V \in \mathcal{A}$. Otherwise, $T \notin \mathcal{S}$, i.e., $T \cap(0, \delta]=\emptyset$ for some $\delta>0$. As $S \in \mathcal{S}$, we can construct $W_{1}, W_{2} \subseteq S$ with $W_{1}, W_{2} \in \mathcal{A}$ and $W_{1} \cap W_{2}=\emptyset$. Then $W_{j} \cup[\delta / 2,1] \in(T, U)_{\prec} \subseteq \mathcal{F}$. Hence also $\emptyset=W_{1} \cap W_{2} \cap(0, \delta / 3] \in \mathcal{F}$, a contradiction.

Since $\prec$ is a dense order, $T \prec W \prec S$ for some closed $W$. Hence also $T \prec V \cup W \prec S$, and $V \cup W \in \mathcal{A}$. Thus $V \cup W \in(T, U)_{\prec} \subseteq \mathcal{X} \subseteq \mathcal{F}$. Hence $\mathcal{X} \subseteq\{S \in \mathcal{A}:(\exists T \prec$ $S)(T \in \mathcal{F})\}$.

$\supseteq:\{S \in \mathcal{A}:(\exists T \prec S)(T \in \mathcal{F})\} \subseteq \mathcal{F}$ and is $\prec$-open: if $T \prec S$ with $T \in \mathcal{F}$, then also $S \in(T,(0,1])_{\prec} \subseteq\{S \in \mathcal{A}:(\exists T \prec S)(T \in \mathcal{F})\}$.

2. As $\mathcal{F}^{\circ} \subseteq \mathcal{F}, \emptyset \notin \mathcal{F}^{\circ}$.

If $U \prec S, V \prec T$ with $U, V \in \mathcal{F}$, then also $U \cap V \prec S \cap T$ with $U \cap V \in \mathcal{F}$.

The other defining properties of an a-filter are immediately checked using part 1.

\section{Theorem 4.7.}

1. For each a-filter $\mathcal{F}$ on $(0,1], \mathcal{F}(I(\mathcal{F}))=\mathcal{F}^{\circ}$.

2. $\left\{\mathcal{F}(I): I \triangleleft \widetilde{\mathbb{K}}_{\mathrm{cnt}}\right\}$ is the set of $\prec$-open a-filters on $(0,1]$.

Proof. First, let $I \triangleleft \widetilde{\mathbb{K}}_{\text {cnt }}$. We show that $\mathcal{F}(I)$ is $\prec$-open:

Let $S \in \mathcal{F}(I)$. Then there exists $x \in I$ such that $x_{\mid S^{c}}$ is invertible. By proposition 3.5, there exists $T \succ S^{c}$ such that $x_{\mid T}$ is invertible. W.l.og. $T$ is open. Then $T^{c} \in \mathcal{F}(I)$ and $T^{c} \prec S$.

In particular, $\mathcal{F}(I(\mathcal{F})) \subseteq \mathcal{F}$ is $\prec$-open, and hence $\mathcal{F}(I(\mathcal{F})) \subseteq \mathcal{F}^{\circ}$.

Conversely, we show that $\mathcal{F}^{\circ} \subseteq \mathcal{F}(I(\mathcal{F}))$ :

Let $S \in \mathcal{F}^{\circ}$. Then there exists $T \prec S$ such that $T \in \mathcal{F}$. By Urysohn's lemma, there exists $x \in \widetilde{\mathbb{K}}_{\text {cnt }}$ such that $x_{\mid T}=0$ and $x_{\mid S^{c}}=1$. Hence $x \in I(\mathcal{F})$ and $S \in \mathcal{F}(I(\mathcal{F}))$.

Finally, if an a-filter $\mathcal{F}$ is $\prec$-open, then $\mathcal{F}=\mathcal{F}^{\circ}=\mathcal{F}(I(\mathcal{F}))$, hence $\mathcal{F}=\mathcal{F}(I)$ for some $I \triangleleft \widetilde{\mathbb{K}}_{\text {cnt }}$.

\section{Theorem 4.8.}

1. For each $I \unlhd \widetilde{\mathbb{K}}_{\mathrm{cnt}}, I(\mathcal{F}(I))=m(I)$.

2. $\{I(\mathcal{F}): \mathcal{F}$ is an a-filter on $(0,1]\}$ is the set of (proper) pure ideals in $\widetilde{\mathbb{K}}_{\mathrm{cnt}}$.

Proof. First, let $\mathcal{F}$ be an a-filter on $(0,1]$. We show that $I(\mathcal{F})$ is pure:

Let $x \in I(\mathcal{F})$. Then there exists $S \in \mathcal{F}$ such that $x_{\mid S}=0$. By proposition 3.5, $x_{\mid T}=0$ for some $T \succ S$. By Urysohn's lemma, there exists $y \in \widetilde{\mathbb{K}}_{\mathrm{cnt}}$ such that $y_{\mid S}=0$, $y_{\mid T^{c}}=1$. Then $(x y)_{\mid T}=0$ and $(x y)_{\mid T^{c}}=x_{\mid T^{c}}$. Hence $x=x y$ and $y \in I(\mathcal{F})$.

In particular, $I(\mathcal{F}(I)) \subseteq I$ is pure for each $I \triangleleft \widetilde{\mathbb{K}}_{\text {cnt }}$, and hence $I(\mathcal{F}(I)) \subseteq m(I)$. Conversely, we show that $m(I) \subseteq I(\mathcal{F}(I))$ for each $I \triangleleft \widetilde{\mathbb{K}}_{\mathrm{cnt}}$ :

Let $x \in m(I)$, i.e., there exists $y \in I$ such that $x=x y$. As $x(1-y)=0$, there exist (by lemma 3.6) closed $S, T \subseteq(0,1]$ with $S \cup T=(0,1]$ such that $x_{\mid S}=0$ and $(1-y)_{\mid T}=0$. Hence $y_{\mid S^{c}}=1$, so $S \in \mathcal{F}(I)$, and $x \in I(\mathcal{F}(I))$.

Finally, if $I \triangleleft \widetilde{\mathbb{K}}_{\text {cnt }}$ is pure, then $I=m(I)=I(\mathcal{F}(I))$, hence $I=I(\mathcal{F})$ for some a-filter $\mathcal{F}$ on $(0,1]$. 


\section{Closed ideals and filters}

We will denote $\bar{I}(\mathcal{F}):=\overline{I(\mathcal{F})}$ (closure in the sharp topology) and $\overline{\mathcal{F}}(I):=\overline{\mathcal{F}(I)}$

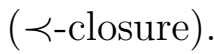

Proposition 5.1. Let $\mathcal{F}$ be an a-filter on $(0,1]$. Then

1. $\overline{\mathcal{F}}=\{S \in \mathcal{A}:(\forall T \succ S, T$ closed $)(T \in \mathcal{F})\}$.

2. $\overline{\mathcal{F}}$ is an a-filter.

Proof. 1. Call $\mathcal{F}^{*}:=\{S \in \mathcal{A}:(\forall T \succ S, T$ closed $)(T \in \mathcal{F})\}$.

$\subseteq: \mathcal{F} \subseteq \mathcal{F}^{*}$ and $\mathcal{F}^{*}$ is $\prec$-closed: if $S \in \mathcal{A} \backslash \mathcal{F}^{*}$, then there exists a closed $T \succ S$ with $T \notin \mathcal{F}$, hence also $(\emptyset, T)_{\prec} \subseteq \mathcal{A} \backslash \mathcal{F}^{*}$.

$\supseteq$ : let $\mathcal{X} \supseteq \mathcal{F}$ be $\prec$-closed. Let $S \in \mathcal{A} \backslash \mathcal{X}$. Then $S \in(T, U)_{\prec} \subseteq \mathcal{A} \backslash \mathcal{X}$ for some $T, U \in \mathcal{A}$. As $\prec$ is a dense order, $S \prec V \prec U$ for some closed $V$, and $V \in(T, U)_{\prec} \subseteq$ $\mathcal{A} \backslash \mathcal{X} \subseteq \mathcal{A} \backslash \mathcal{F}$. Thus $S \notin \mathcal{F}^{*}$. Hence $\mathcal{F}^{*} \subseteq \mathcal{X}$.

2. $\emptyset \notin \overline{\mathcal{F}}$, since $\emptyset \notin \mathcal{A}$.

Let $S_{1}, S_{2} \in \overline{\mathcal{F}}$ and let $T \succ S_{1} \cap S_{2}$. Let

$$
\begin{aligned}
& U_{1}=\left\{\varepsilon \in(0,1]: d\left(\varepsilon, S_{1}\right)<d\left(\varepsilon, S_{2}\right)\right\} \\
& U_{2}=\left\{\varepsilon \in(0,1]: d\left(\varepsilon, S_{2}\right)<d\left(\varepsilon, S_{1}\right)\right\} .
\end{aligned}
$$

Let $V_{1}:=U_{1} \cup T^{\circ}$ and $V_{2}:=U_{2} \cup T^{\circ}$. Then $S_{1}=\left(S_{1} \backslash S_{2}\right) \cup\left(S_{1} \cap S_{2}\right) \subseteq U_{1} \cup T^{\circ}=V_{1}$ since $S_{2}$ is closed. Since $V_{1}$ is open, $S_{1} \prec V_{1}$. Hence $V_{1} \in \mathcal{F}$. Similarly, $V_{2} \in \mathcal{F}$. As $V_{1} \cap V_{2} \subseteq T, T \in \mathcal{F}$. We conclude that $S_{1} \cap S_{2} \in \overline{\mathcal{F}}$.

The other defining properties of an a-filter are immediately checked using part 1 .

Corollary 5.2. If $\mathcal{F}$ is an a-filter on $(0,1]$, then $\overline{\mathcal{F} \circ}=\overline{\mathcal{F}}$.

Proof. $\supseteq$ : since $\mathcal{F}^{\circ} \subseteq \mathcal{F}$.

$\subseteq$ : it suffices to show that $\mathcal{F} \subseteq \overline{\mathcal{F}^{\circ}}$. Let $S \in \mathcal{F}$. Let $T \subseteq(0,1]$ be closed such that $T \succ S$. Then $T \in \mathcal{F}^{\circ}$. Hence $S \in \overline{\mathcal{F}^{\circ}}$.

Theorem 5.3. Let $\mathcal{F}$ be an a-filter. Then

$$
\bar{I}(\mathcal{F})=\left\{x \in \widetilde{\mathbb{K}}_{\mathrm{cnt}}:(\forall S \in \mathcal{A})\left(x_{\mid S^{c}} \text { invertible } \Rightarrow S \in \mathcal{F}\right)\right\} .
$$

Proof. Call $I^{+}(\mathcal{F}):=\left\{x \in \widetilde{\mathbb{K}}_{\mathrm{cnt}}:(\forall S \in \mathcal{A})\left(x_{\mid S^{c}}\right.\right.$ invertible $\left.\left.\Rightarrow S \in \mathcal{F}\right)\right\}$.

We first show that $I^{+}(\mathcal{F})$ is closed:

If $a \in \widetilde{\mathbb{K}}_{\text {cnt }} \backslash I^{+}(\mathcal{F})$, then there exists $S \in \mathcal{A} \backslash \mathcal{F}$ such that $a_{\mid S^{c}}$ is invertible. By lemma 3.4, $x_{\mid S^{c}}$ is invertible for each $x$ in a certain neighborhood of $a$. Then such $x \notin I^{+}(\mathcal{F})$, too. Hence $\widetilde{\mathbb{K}}_{\text {cnt }} \backslash I^{+}(\mathcal{F})$ is open.

We now show that $I(\mathcal{F}) \subseteq I^{+}(\mathcal{F})$ :

Let $x \in I(\mathcal{F})$. Then $x_{\mid S}=0$ for some $S \in \mathcal{F}$. Let $T \in \mathcal{A}$ such that $x_{\mid T^{c}}$ is invertible. Then $S \cap(0, \delta) \backslash T=\emptyset$ for some $\delta>0$, for otherwise, $0 \in \overline{S \backslash T}$ and $x_{\mid S \backslash T}=0$ and $x_{\mid S \backslash T}$ is invertible, a contradiction. Hence $S \cap(0, \delta) \subseteq T$, and $T \in \mathcal{F}$. Thus $x \in I^{+}(\mathcal{F})$. Finally, we show that $I^{+}(\mathcal{F}) \subseteq \bar{I}(\mathcal{F})$ :

Let $x=\left[x_{\varepsilon}\right] \in I^{+}(\mathcal{F})$. Consider the sets $L_{n}:=\left\{\varepsilon:\left|x_{\varepsilon}\right|>\varepsilon^{n}\right\}$. As $x_{\mid L_{n}}$ is invertible, $L_{n}^{c} \in \mathcal{F}$, for each $n \in \mathbb{N}$. Further, $L_{n} \prec L_{n+1}$ for each $n \in \mathbb{N}$. By Urysohn's 
lemma, there exist $y_{n} \in \widetilde{\mathbb{K}}_{\mathrm{cnt}}$ such that $y_{n \mid L_{n}}=1$ and $y_{n \mid L_{n+1}^{c}}=0$ and $0 \leq y_{n} \leq 1$. Then $\left|x y_{n}-x\right|_{\mid L_{n}}=0$ and $\left|x y_{n}-x\right|_{\mid L_{n}^{c}} \leq|x|_{\mid L_{n}^{c}} \leq \rho^{n}$. Hence $\left|x y_{n}-x\right| \leq \rho^{n}$, and $\lim _{n \rightarrow \infty} x y_{n}=x$. As $\left(x y_{n}\right)_{\mid L_{n+1}^{c}}=0, x y_{n} \in I(\mathcal{F})$, for each $n$.

Corollary 5.4. Let $I \triangleleft \widetilde{\mathbb{K}}_{\mathrm{cnt}}$. Then $I(\mathcal{F}(I)) \subseteq I \subseteq \bar{I}(\mathcal{F}(I))$ and $\bar{I}=\overline{m(I)}$.

Proof. $I \subseteq \bar{I}(\mathcal{F}(I))$ : let $x \in I$. Let $S \in \mathcal{A}$ such that $x_{\mid S^{c}}$ is invertible. Then $S \in \mathcal{F}(I)$. Hence by theorem [5.3, $x \in \bar{I}(\mathcal{F}(I))$.

By proposition 4.5, $I(\mathcal{F}(I)) \subseteq I$. Hence $\bar{I}=\bar{I}(\mathcal{F}(I))=\overline{m(I)}$ by theorem 4.8.

Theorem 5.5. Let $I \triangleleft \widetilde{\mathbb{K}}_{\mathrm{cnt}}$. Then

$$
\overline{\mathcal{F}}(I)=\left\{S \in \mathcal{A}:\left(\forall x \in \widetilde{\mathbb{K}}_{\mathrm{cnt}}\right)\left(x_{\mid S}=0 \Rightarrow x \in I\right)\right\} .
$$

Proof. Call $\mathcal{F}^{+}(I):=\left\{S \in \mathcal{A}:\left(\forall x \in \widetilde{\mathbb{K}}_{\mathrm{cnt}}\right)\left(x_{\mid S}=0 \Rightarrow x \in I\right)\right\}$.

We show that $\mathcal{F}^{+}(I)$ is closed:

Let $S \in \overline{\mathcal{F}^{+}}(I)$, i.e. $S \in \mathcal{A}$ and $T \in \mathcal{F}^{+}(I)$, for each closed $T \succ S$. Let $x \in \widetilde{\mathbb{K}}_{\text {cnt }}$ such that $x_{\mid S}=0$. By lemma 3.5, there exists $T \succ S$ such that $x_{\mid T}=0$. W.l.o.g, $T$ is closed. Thus $x \in I$. Hence $S \in \mathcal{F}^{+}(I)$.

We now show that $\mathcal{F}(I) \subseteq \mathcal{F}^{+}(I)$ :

Let $S \in \mathcal{F}(I)$. Then there exists $a \in I$ such that $a_{\mid S^{c}}=1$. Now let $x \in \widetilde{\mathbb{K}}_{\text {cnt }}$ such that $x_{\mid S}=0$. Then $x_{\mid T}=0$ for some $T \succ S$. By Urysohn's lemma, there exists $y \in \widetilde{\mathbb{K}}_{\text {cnt }}$ with $y_{\mid S}=0$ and $y_{\mid T^{c}}=1$. Then $(x y a)_{\mid T}=x_{\mid T}=0$ and $(x y a)_{\mid T^{c}}=x_{\mid T^{c}}$. Hence $x=x y a \in I$.

Finally, we show that $\mathcal{F}^{+}(I) \subseteq \overline{\mathcal{F}}(I)$ :

Let $S \in \mathcal{F}^{+}(I)$ and let $T \succ S$ be closed. By Urysohn's lemma, there exists $y \in \widetilde{\mathbb{K}}_{\text {cnt }}$ such that $y_{\mid S}=0$ and $y_{\mid T^{c}}=1$. As $S \in \mathcal{F}^{+}(I), y \in I$. Hence $T \in \mathcal{F}(I)$.

Theorem 5.6. If $I, J \triangleleft \widetilde{\mathbb{K}}_{\text {cnt }}$, then $\mathcal{F}(I)=\mathcal{F}(J) \Longleftrightarrow m(I)=m(J) \Longleftrightarrow \bar{I}=\bar{J}$.

Proof. 1. If $\mathcal{F}(I)=\mathcal{F}(J)$, then $m(I)=I(\mathcal{F}(I))=I(\mathcal{F}(J))=m(J)$ by theorem 4.8,

2. If $m(I)=m(J)$, then $\bar{I}=\overline{m(I)}=\overline{m(J)}=\bar{J}$ by corollary 5.4 .

3. Let $S \in \mathcal{F}(\bar{I})$. Let $E:=\left\{x \in \widetilde{\mathbb{K}}_{\mathrm{cnt}}: x_{\mid S^{c}}\right.$ is invertible $\}$. Then $\bar{I} \cap E \neq \emptyset$. By lemma 3.4, $E$ is open, hence also $I \cap E \neq \emptyset$, i.e., $S \in \mathcal{F}(I)$.

Hence, if $\bar{I}=\bar{J}$, then $\mathcal{F}(I)=\mathcal{F}(\bar{I})=\mathcal{F}(\bar{J})=\mathcal{F}(J)$.

Corollary 5.7. If $I \triangleleft \widetilde{\mathbb{K}}_{\mathrm{cnt}}$, then $m(\bar{I})=m(I)$.

Theorem 5.8. Let $\mathcal{F}_{1}, \mathcal{F}_{2}$ be a-filters on $(0,1]$. Then $I\left(\mathcal{F}_{1}\right)=I\left(\mathcal{F}_{2}\right) \Longleftrightarrow \mathcal{F}_{1}^{\circ}=$ $\mathcal{F}_{2}^{\circ} \Longleftrightarrow \overline{\mathcal{F}_{1}}=\overline{\mathcal{F}_{2}}$.

Proof. 1. If $I\left(\mathcal{F}_{1}\right)=I\left(\mathcal{F}_{2}\right)$, then $\mathcal{F}_{1}^{\circ}=\mathcal{F}\left(I\left(\mathcal{F}_{1}\right)\right)=\mathcal{F}\left(I\left(\mathcal{F}_{2}\right)\right)=\mathcal{F}_{2}^{\circ}$ by theorem 4.7.

2. If $\mathcal{F}_{1}^{\circ}=\mathcal{F}_{2}^{\circ}$, then $\overline{\mathcal{F}_{1}}=\overline{\mathcal{F}_{1}^{\circ}}=\overline{\mathcal{F}_{2}^{\circ}}=\overline{\mathcal{F}_{2}}$ by corollary 5.2 .

3. Let $x \in I(\overline{\mathcal{F}})$. Then $x_{\mid S}=0$ for some $S \in \overline{\mathcal{F}}$. By proposition 3.5, there exists $T \succ S$ (w.l.o.g. $T$ closed) such that $x_{\mid T}=0$. So $T \in \mathcal{F}$, and $x \in I(\mathcal{F})$.

Hence, if $\overline{\mathcal{F}_{1}}=\overline{\mathcal{F}_{2}}$, then $I\left(\mathcal{F}_{1}\right)=I\left(\overline{\mathcal{F}_{1}}\right)=I\left(\overline{\mathcal{F}_{2}}\right)=I\left(\mathcal{F}_{2}\right)$.

Corollary 5.9. If $\mathcal{F}$ is an a-filter on $(0,1]$, then $(\overline{\mathcal{F}})^{\circ}=\mathcal{F}^{\circ}$. 


\section{Maximal and prime ideals and filters}

Definition 6.1. An a-filter $\mathcal{F}$ on $(0,1]$ is called prime if for each $S, T \in \mathcal{A}$ with $S \cup T \in \mathcal{F}$, either $S \in \mathcal{F}$ or $T \in \mathcal{F}$.

An a-filter $\mathcal{F}$ on $(0,1]$ is called pseudoprime if for each $S, T \in \mathcal{A}$ with $S^{\circ} \cup T^{\circ}=(0,1]$, either $S \in \mathcal{F}$ or $T \in \mathcal{F}$.

Remark 6.2. 1. In the definition of (pseudo)prime a-filter, we may also ask the condition for each closed $S, T \subseteq(0,1]$ (instead of for each $S, T \in \mathcal{A}$ only). For, if $S \notin \mathcal{A}$, then $S \notin \mathcal{S}$, i.e., $(0, \delta] \cap S=\emptyset$ for some $\delta>0$. Hence $(S \cup T) \cap(0, \delta] \subseteq T$. So if $S \cap T \in \mathcal{F}$, then also $T \in \mathcal{F}$. The case $T \notin \mathcal{A}$ is symmetric.

2. An a-filter $\mathcal{F}$ on $(0,1]$ is prime if and only if for each $S, T \in \mathcal{A}$ with $S \cup T=(0,1]$, either $S \in \mathcal{F}$ or $T \in \mathcal{F}$. For, if $\mathcal{F}$ satisfies the latter condition and $S \cup T \in \mathcal{F}$, we consider

$$
U:=\{\varepsilon \in(0,1]: d(\varepsilon, S) \leq d(\varepsilon, T)\} \quad \text { and } \quad V:=\{\varepsilon \in(0,1]: d(\varepsilon, T) \leq d(\varepsilon, S)\} .
$$

Then $U, V$ are closed with $U \cup V=(0,1]$. Hence $U \in \mathcal{F}$ or $V \in \mathcal{F}$. If $U \in \mathcal{F}$, then also $(S \cup T) \cap U \in \mathcal{F}$. As $(S \cup T) \cap U \subseteq S$, also $S \in \mathcal{F}$. The case $V \in \mathcal{F}$ is symmetric. This motivates our (less obvious) definition of pseudoprime a-filter.

Lemma 6.3. Let $S, T, U \subseteq(0,1]$ be open and nonempty with $\bar{U} \subseteq S \cup T$. Then there exist $V \prec S$ and $W \prec T$ such that $U \subseteq V \cup W$.

Proof. Let

$$
\begin{aligned}
V & :=\left\{\varepsilon \in(0,1]: \max \left(d(\varepsilon, U), d\left(\varepsilon, T^{c}\right)\right) \leq d\left(\varepsilon, S^{c}\right)\right\} \\
W & :=\left\{\varepsilon \in(0,1]: \max \left(d(\varepsilon, U), d\left(\varepsilon, S^{c}\right)\right) \leq d\left(\varepsilon, T^{c}\right)\right\} .
\end{aligned}
$$

If $\varepsilon \in V \backslash S$, then $\varepsilon \in T^{c} \cap \bar{U} \subseteq T^{c} \cap(S \cup T) \subseteq S$. Hence $V \subseteq S$. As $V$ is closed and $S$ is open, also $V \prec S$. Similarly $W \prec T$.

Further, let $\varepsilon \in U$. Then either $d\left(\varepsilon, T^{c}\right) \leq d\left(\varepsilon, S^{c}\right)$ (hence $\left.\varepsilon \in V\right)$ or $d\left(\varepsilon, S^{c}\right) \leq d\left(\varepsilon, T^{c}\right)$ (hence $\varepsilon \in W$ ). So $U \subseteq V \cup W$.

Lemma 6.4. Let $\mathcal{F}$ be a pseudoprime a-filter on $(0,1]$. Then $I(\mathcal{F})$ is pseudoprime.

Proof. Let $x y=0$. By lemma 3.6, there exist closed $T, U$ with $T^{\circ} \cup U^{\circ}=(0,1]$ such that $x_{\mid T}=0$ and $y_{\mid U}=0$. As $\mathcal{F}$ is pseudoprime, $T \in \mathcal{F}$ or $U \in \mathcal{F}$. Hence $x \in I(\mathcal{F})$ or $y \in I(\mathcal{F})$.

Lemma 6.5. Let $I \triangleleft \widetilde{\mathbb{K}}_{\mathrm{cnt}}$ be pseudoprime. Then $\mathcal{F}(I)$ is pseudoprime.

Proof. Let $S, T \in \mathcal{A}$ with $S^{\circ} \cup T^{\circ}=(0,1]$. Let $V \prec S^{\circ}$ and $W \prec T^{\circ}$ such that $V \cup W=$ $(0,1]$ (lemma 6.3 with $U=(0,1])$. By Urysohn's lemma, there exist $x, y \in \widetilde{\mathbb{K}}_{\text {cnt }}$ such that $x_{\mid V}=0, x_{\mid S^{c}}=1, y_{\mid W}=0$ and $y_{\mid T^{c}}=1$. Then $x y=0$. As $I$ is pseudoprime, $x \in I$ or $y \in I$. Hence $S \in \mathcal{F}(I)$ or $T \in \mathcal{F}(I)$.

Lemma 6.6. Every closed ideal $I \triangleleft \widetilde{\mathbb{K}}_{\mathrm{cnt}}$ is radical.

Proof. Let $S \in \mathcal{F}(\sqrt{I})$. Then there exists $x \in \widetilde{\mathbb{K}}_{\text {cnt }}$ and $n \in \mathbb{N}$ with $x^{n} \in I$ and $x_{\mid S^{c}}=1$. Then also $x^{n}{ }_{\mid S^{c}}=1$, hence $S \in \mathcal{F}(I)$. Thus $\mathcal{F}(\sqrt{I})=\mathcal{F}(I)$. By theorem 5.6, $I \subseteq \sqrt{I} \subseteq \overline{\sqrt{I}}=\bar{I}=I$. 
Proposition 6.7. Let $I \triangleleft \widetilde{\mathbb{K}}_{\mathrm{cnt}}$. Then the following are equivalent:

1. I is pseudoprime

2. the set of ideals containing I is totally ordered (for $\subseteq$ )

3. I is irreducible

4. $\sqrt{I}$ is prime

5. $\mathcal{F}(I)$ is pseudoprime.

For $\widetilde{\mathbb{K}}_{\mathrm{cnt}}=\widetilde{\mathbb{R}}_{\mathrm{cnt}}$, this is still equivalent with

6. $\widetilde{\mathbb{R}}_{\mathrm{cnt}} / I$ is totally ordered.

Proof. $1 \Rightarrow 6$ (for $\left.\widetilde{\mathbb{K}}_{\text {cnt }}=\widetilde{\mathbb{R}}_{\text {cnt }}\right)$ : let $a \in \widetilde{\mathbb{R}}_{\text {cnt }}$. Since $a^{2}=|a|^{2}$, we have $(a-|a|)(a+|a|)=$ 0 . As $I$ is pseudoprime, $a-|a| \in I$ or $a+|a| \in I$. As $\widetilde{\mathbb{R}}_{\text {cnt }}$ is an l-ring, it follows that $a+I \geq 0$ or $-a+I \geq 0$ in $\widetilde{\mathbb{R}}_{\text {cnt }} / I$ (cf. [7, Thm. 5.3]).

$6 \Rightarrow 2$ (for $\widetilde{\mathbb{K}}_{\mathrm{cnt}}=\widetilde{\mathbb{R}}_{\mathrm{cnt}}$, cf. [8, 4.1]): the map $J \mapsto J / I$ is an order preserving bijection between the $\left(l\right.$-)ideals of $\widetilde{\mathbb{R}}_{\text {cnt }}$ containing $I$ and the $l$-ideals of $\widetilde{\mathbb{R}}_{\text {cnt }} / I$. As in any totally ordered ring, the $l$-ideals in $\widetilde{\mathbb{R}}_{\text {cnt }} / I$ are totally ordered.

$1 \Rightarrow 2$ (for $\widetilde{\mathbb{K}}_{\mathrm{cnt}}=\widetilde{\mathbb{C}}_{\mathrm{cnt}}$ ): by the bijective correspondence of ideals in $\widetilde{\mathbb{R}}_{\mathrm{cnt}}$ and in $\widetilde{\mathbb{C}}_{\mathrm{cnt}}$ (section 2).

$2 \Rightarrow 3$ : let $K=I \cap J$. Either $I \subseteq J$ or $J \subseteq I$, whence $K=I$ or $K=J$.

$3 \Rightarrow 1$ : as in any commutative $l$-ring with 1 in which every ideal is an $l$-ideal, the irreducibility of $I \unlhd \widetilde{\mathbb{R}}_{\text {cnt }}$ is equivalent with: for any $x, y \in \widetilde{\mathbb{R}}_{\text {cnt }}, x \widetilde{\mathbb{R}}_{\text {cnt }} \cap y \widetilde{\mathbb{R}}_{\text {cnt }} \subseteq I$ implies $x \in I$ or $y \in I$ [2, Prop. 8.4.1]. So let $x, y \in \widetilde{\mathbb{R}}_{\text {cnt }}$ with $x y=0$. By lemma 3.6, there exist open $T, U$ with $T \cup U=(0,1]$ such that $x_{\mid T}=0$ and $y_{\mid U}=0$. Let $z \in x \widetilde{\mathbb{R}}_{\text {cnt }} \cap y \widetilde{\mathbb{R}}_{\text {cnt }}$. Then $z_{\mid T}=z_{\mid U}=0$, hence $z=0$. In particular, $x \widetilde{\mathbb{R}}_{\text {cnt }} \cap y \widetilde{\mathbb{R}}_{\text {cnt }} \subseteq I$, and hence $x \in I$ or $y \in I$. The bijective correspondence of ideals in $\widetilde{\mathbb{R}}_{\text {cnt }}$ and $\widetilde{\mathbb{C}}_{\text {cnt }}$ yields the result for $\widetilde{\mathbb{C}}_{\text {cnt }}$.

$2 \Rightarrow 4$ : the intersection of a chain of prime ideals is prime, hence $\sqrt{I}=\bigcap_{I \subseteq P, P \text { prime }} P$ is prime.

$4 \Rightarrow 5$ : by lemma 6.5, $\mathcal{F}(\sqrt{I})$ is pseudoprime. By the proof of lemma 6.6, $\mathcal{F}(I)=$ $\mathcal{F}(\sqrt{I})$.

$5 \Rightarrow 1$ : by lemma 6.4, $m(I)=I(\mathcal{F}(I))$ is pseudoprime. Hence $I \supseteq I(\mathcal{F}(I))$ is also pseudoprime.

Theorem 6.8. Let $I \triangleleft \widetilde{\mathbb{K}}_{\mathrm{cnt}}$. Then $I$ is prime iff $I$ is pseudoprime and radical.

Proof. $\Rightarrow$ : as $I$ is prime, $\sqrt{I}=\bigcap_{I \subseteq P, P \text { prime }} P=I$.

$\Leftarrow: I=\sqrt{I}$ is prime by proposition 6.7,

Lemma 6.9. Every pure ideal $I \triangleleft \widetilde{\mathbb{K}}_{\mathrm{cnt}}$ is radical.

Proof. Let $x^{n} \in I$ for some $x \in \widetilde{\mathbb{K}}_{\mathrm{cnt}}$ and $n \in \mathbb{N}$. As $I=m(I)=I(\mathcal{F}(I))$, there exists $S \in \mathcal{F}(I)$ such that $x^{n}{ }_{\mid S}=0$. Hence also $x_{\mid S}=0$, and $x \in I(\mathcal{F}(I))=I$.

Proposition 6.10. For $I \triangleleft \widetilde{\mathbb{K}}_{\mathrm{cnt}}$, the following are equivalent: 


\section{I is pseudoprime \\ 2. $m(I)$ is prime \\ 3. I contains a prime ideal.}

Proof. $1 \Rightarrow 2$ : by lemmas 6.4 and 6.5, $m(I)=I(\mathcal{F}(I))$ is pseudoprime. By lemma 6.9, $m(I)$ is radical. Hence $m(I)$ is prime.

$2 \Rightarrow 3: m(I) \subseteq I$.

$3 \Rightarrow 1$ : if $P \subseteq I$ is prime and $x y=0$, then $x y \in P$, so $x \in P \subseteq I$ or $y \in P \subseteq I$.

Proposition 6.11. Let $\mathcal{F}$ be an a-filter on $(0,1]$. Then the following are equivalent:

1. $\mathcal{F}$ is pseudoprime

2. $I(\mathcal{F})$ is pseudoprime

3. $I(\mathcal{F})$ is prime.

Proof. $1 \Rightarrow 2$ : by lemma 6.4, $I(\mathcal{F})$ is pseudoprime.

$2 \Rightarrow 3$ : as $I(\mathcal{F})$ is pure, $I(\mathcal{F})$ is radical (lemma 6.9). By theorem 6.8, $I(\mathcal{F})$ is prime. $3 \Rightarrow 1$ : by lemma 6.5, $\mathcal{F}(I(\mathcal{F}))$ is pseudoprime. As $\mathcal{F}(I(\mathcal{F})) \subseteq \mathcal{F}$, also $\mathcal{F}$ is pseudoprime.

We now consider maximal ideals and a-filters:

Theorem 6.12. Let $\mathcal{F}$ be an a-filter.

1. if $\mathcal{F}$ is pseudoprime, then $\overline{\mathcal{F}}$ is maximal.

2. $\mathcal{F}$ is maximal if and only if $\mathcal{F}$ is prime and $\prec$-closed.

Proof. 1. Suppose $\overline{\mathcal{F}} \subsetneq \mathcal{F}^{\prime}$ for some a-filter $\mathcal{F}^{\prime}$. Let $S \in \mathcal{F}^{\prime} \backslash \overline{\mathcal{F}}$. Then there exists a closed $T \succ S$ such that $T \notin \mathcal{F}$. As $\prec$ is a dense order, there exists an open $V$ with $S \prec V \prec T$. Since $T^{\circ} \cup\left(V^{c}\right)^{\circ}=(0,1]$ and $\mathcal{F}$ is pseudoprime, $V^{c} \in \mathcal{F}$. But then $\emptyset=S \cap V^{c} \in \mathcal{F}^{\prime}$, a contradiction.

2. $\Rightarrow$ : we show that $\mathcal{F}$ is closed: as $\mathcal{F} \subseteq \overline{\mathcal{F}}$, and $\overline{\mathcal{F}}$ is an a-filter, $\mathcal{F}=\overline{\mathcal{F}}$ by maximality. Further, we show that $\mathcal{F}$ is prime: let $S, T \in \mathcal{A}$ such that $S \cup T \in \mathcal{F}$. Suppose there exists $U \in \mathcal{F}$ such that $U \cap S=\emptyset$ and there exists $V \in \mathcal{F}$ such that $V \cap T=\emptyset$. Then $\emptyset=(U \cap V) \cap(S \cup T) \in \mathcal{F}$, a contradiction. We may thus assume that $U \cap S \neq \emptyset$, for each $U \in \mathcal{F}$. (The case $U \cap T \neq \emptyset$, for each $U \in \mathcal{F}$ is similar.) Then $\emptyset \notin \mathcal{F}^{\prime}:=\{U \subseteq(0,1]$ closed: $(\exists V \in \mathcal{F})(S \cap V \subseteq U)\}$. As $\mathcal{F}^{\prime}$ is an a-filter, $\mathcal{F}=\mathcal{F}^{\prime}$ by maximality. Hence $S \in \mathcal{F}$.

2 . $\Leftarrow$ : by part $1, \mathcal{F}=\overline{\mathcal{F}}$ is maximal.

Theorem 6.13. Let $I \triangleleft \widetilde{\mathbb{K}}_{\mathrm{cnt}}$.

1. if $I$ is pseudoprime, then $\bar{I}$ is maximal.

2. I is maximal if and only if $I$ is prime and closed. 
Proof. 1. By proposition 6.7, $\mathcal{F}(I)=\mathcal{F}(\bar{I})$ is pseudoprime. Thus by theorem 6.12, $\overline{\mathcal{F}}(\bar{I})$ is maximal. Now let $\bar{I} \subseteq J \triangleleft \widetilde{\mathbb{K}}_{\text {cnt }}$. Then $\overline{\mathcal{F}}(\bar{I}) \subseteq \overline{\mathcal{F}}(J)$, and hence $\overline{\mathcal{F}}(\bar{I})=\overline{\mathcal{F}}(J)$ by maximality. Hence also $m(\bar{I})=I(\mathcal{F}(\bar{I}))=I(\overline{\mathcal{F}}(\bar{I}))=I(\overline{\mathcal{F}}(J))=I(\mathcal{F}(J))=m(J)$, and hence $J \subseteq \bar{J}=\bar{I}$ by theorem 5.6 .

2. $\Rightarrow$ : let $E$ denote the set of invertible elements in $\widetilde{\mathbb{K}}_{\mathrm{cnt}}$. As $I$ is a proper ideal, $I \cap E=\emptyset$. As $E$ is open, also $\bar{I} \cap E=\emptyset$. Hence $\bar{I}$ is proper, and $I=\bar{I}$ by maximality. Maximal ideals are prime in any commutative ring with 1.

$\Leftarrow$ : by part $1, I=\bar{I}$ is maximal.

\section{Corollary 6.14.}

1. The set of minimal prime ideals in $\widetilde{\mathbb{K}}_{\text {cnt }}$ equals $\{I(\mathcal{F}): \mathcal{F}$ is a max. a-filter on $(0,1]\}=\{I(\mathcal{F}): \mathcal{F}$ is a pseudoprime a-filter on $(0,1]\}$.

2. The set of maximal ideals in $\widetilde{\mathbb{K}}_{\mathrm{cnt}}$ equals

$\{\bar{I}(\mathcal{F}): \mathcal{F}$ is a max. a-filter on $(0,1]\}=\{\bar{I}(\mathcal{F}): \mathcal{F}$ is a pseudoprime a-filter on $(0,1]\}$.

Proof. 1.(a) Let $I \triangleleft \widetilde{\mathbb{K}}_{\mathrm{cnt}}$ be a minimal prime. Then $\mathcal{F}(I)$ is pseudoprime, and $I(\mathcal{F}(I)) \subseteq I$ is a prime ideal. By minimality, $I=I(\mathcal{F}(I))=I(\overline{\mathcal{F}}(I))$ and $\overline{\mathcal{F}}(I)$ is maximal.

(b) Let $\mathcal{F}$ be a pseudoprime a-filter on $(0,1]$. Then $I(\mathcal{F})$ is prime by proposition 6.11, If $P \triangleleft \widetilde{\mathbb{K}}_{\text {cnt }}$ is prime with $P \subseteq I(\mathcal{F})$, then $\mathcal{F}(P) \subseteq \mathcal{F}(I(\mathcal{F})) \subseteq \mathcal{F}$, and hence $\overline{\mathcal{F}}(P) \subseteq \overline{\mathcal{F}}$. As $P$ is prime, $\mathcal{F}(P)$ is pseudoprime, and hence $\overline{\mathcal{F}}(P)$ is maximal by theorem 6.12 . Hence $\overline{\mathcal{F}}(P)=\overline{\mathcal{F}}$. Consequently, $P \supseteq I(\mathcal{F}(P))=I(\overline{\mathcal{F}}(P))=I(\overline{\mathcal{F}})=I(\mathcal{F})$.

2.(a) Let $I \triangleleft \widetilde{\mathbb{K}}_{\text {cnt }}$ be maximal. Then $I$ is pseudoprime, hence $\mathcal{F}(I)$ is pseudoprime, and thus $\overline{\mathcal{F}}(I)$ is maximal. Further, $I=\bar{I}=\overline{m(I)}=\bar{I}(\mathcal{F}(I))=\bar{I}(\overline{\mathcal{F}}(I))$.

(b) Let $\mathcal{F}$ be a pseudoprime a-filter on $(0,1]$. Then $I(\mathcal{F})$ is pseudoprime, hence $\bar{I}(\mathcal{F})$ is maximal.

Proposition 6.15. Let $I \triangleleft \widetilde{\mathbb{K}}$. Then $\bar{I}=\bigcap_{M}^{I \subseteq M} \underset{\text { maximal }}{I \text {. }}$.

In particular, an ideal $I \triangleleft \widetilde{\mathbb{K}}$ is closed iff it is an intersection of maximal ideals.

Proof. $\subseteq$ : by theorem 6.13, maximal ideals are closed.

: let $x \notin \bar{I}=\overline{m(I)}=\bar{I}(\mathcal{F}(I))$ (corollary 5.4). By theorem 5.3, there exists $S \in$ $\mathcal{A} \backslash \mathcal{F}(I)$ such that $x_{\mid S^{c}}$ is invertible. Let $E:=\left\{x \in \widetilde{\mathbb{K}}_{\mathrm{cnt}}: x_{\mid S^{c}}\right.$ is invertible $\}$. As $E$ is closed under multiplication and $E \cap I=\emptyset$, there exists a prime $P \triangleleft \widetilde{\mathbb{K}}_{\text {cnt }}$ such that $I \subseteq P$ and $E \cap P=\emptyset$ (e.g., [7, 0.16]). As $E$ is open (lemma 3.4), also $E \cap \bar{P}=\emptyset$. In particular, $\bar{P}$ is maximal and $x \notin \bar{P}$.

Remark 6.16. In the previous, we showed that maximal ideals of $\widetilde{\mathbb{K}}_{\text {cnt }}$ are in bijective correpondence with maximal a-filters, which are in bijective correspondence with points of $\beta(0,1] \backslash(0,1]$, where $\beta(0,1]$ denotes the Stone-Čech compactification of $(0,1]$ (cf. [7, 6.5]).

\section{$7 \quad$ Rapid a-filters}

Definition 7.1. An a-filter $\mathcal{F}$ is called rapid if for each sequence $\left(S_{n}\right)_{n}$ in $\mathcal{F}$ with $S_{1} \succ S_{2} \succ \ldots$, there exists $T \in \mathcal{F}$ such that $T \backslash S_{n} \notin \mathcal{S}$. 
Theorem 7.2. Let $\mathcal{F}$ be an a-filter. Then $I(\mathcal{F})$ is closed iff $\mathcal{F}$ is rapid.

Proof. $\Leftarrow$ : let $a \in \bar{I}(\mathcal{F})$ with continuous representative $\left(a_{\varepsilon}\right)_{\varepsilon}$. For each $n \in \mathbb{N}$, let $S_{n}:=\left\{\varepsilon \in(0,1]:\left|a_{\varepsilon}\right| \leq \varepsilon^{n}\right\}$. By theorem [5.3, $S_{n} \in \mathcal{F}$, and also $S_{1} \succ S_{2} \succ \ldots$ As $\mathcal{F}$ is rapid, there exists $T \in \mathcal{F}$ such that $T \backslash S_{n} \notin \mathcal{S}$. Hence $|a|_{\mid T} \leq \rho^{n}$, for each $n \in \mathbb{N}$, i.e., $a_{\mid T}=0$. Hence $a \in I(\mathcal{F})$.

$\Rightarrow$ : let $S_{n} \in \mathcal{F}$, and also $S_{1} \succ S_{2} \succ \ldots$ By Urysohn's lemma, there exist $\phi_{n} \in \mathcal{C}((0,1])$ such that $0 \leq \phi_{n} \leq \varepsilon^{n}, \phi_{n \mid S_{n+1}}=0$ and $\phi_{n \mid S_{n}^{c}}=\varepsilon^{n}$. Let $\phi:=\sum_{n=1}^{\infty} \phi_{n}$ on $(0,1 / 2]$. By uniform convergence, $\phi$ is continuous and $\varepsilon^{n+1} \leq \phi(\varepsilon) \leq \varepsilon^{n}+\varepsilon^{n+1}+\cdots \leq 2 \varepsilon^{n}$ on $(0,1 / 2] \cap S_{n} \backslash S_{n+1}$. Extend $\phi$ to a continuous map on $(0,1]$. Then $a:=[\phi(\varepsilon)] \in \widetilde{\mathbb{K}}_{\mathrm{cnt}}$. Let $T \in \mathcal{A}$ be such that $a_{\mid T^{c}}$ is invertible. Then there exists $n \in \mathbb{N}$ such that $|\phi(\varepsilon)|>$ $2 \varepsilon^{n}$ for $\varepsilon \in T^{c} \cap(0, \delta]$ (some $\left.0<\delta \leq 1 / 2\right)$. Hence $S_{n} \cap(0, \delta] \subseteq T$, and $T \in \mathcal{F}$. By theorem [5.3, $a \in \bar{I}(\mathcal{F})=I(\mathcal{F})$. Thus there exists $T \in \mathcal{F}$ such that $a_{\mid T}=0$.

Let $n \in \mathbb{N}$. Then $|\phi(\varepsilon)|<\varepsilon^{n}$ for each $\varepsilon \in(0, \delta] \cap T$ (some $\left.0<\delta \leq 1 / 2\right)$. Hence $(0, \delta] \cap T \backslash S_{n}=\emptyset$.

Remark 7.3. Recall that a filter $\mathcal{F}$ of subsets of $\mathbb{N}$ is called rapid if for any decreasing sequence $\left(S_{n}\right)_{n}$ in $\mathcal{F}$, there exists $S \in \mathcal{F}$ such that $S \backslash S_{n}$ is finite for every $n \in \mathbb{N}$. A free ultrafilter $\mathcal{U}$ of subsets of $\mathbb{N}$ is called weakly selective (or $\delta$-stable or P-point of $\beta \mathbb{N} \backslash \mathbb{N}$ ) if for each sequence $\left(S_{n}\right)_{n}$ in $\mathcal{U}$, there exists $S \in \mathcal{U}$ such that $S \backslash S_{n}$ is finite for each $n \in \mathbb{N}$. There exist weakly selective free ultrafilters if we assume the continuum hypothesis [11, 6] (in fact, it satisfies to assume weaker axioms, e.g. ZFC+Martin's axiom [3, §4]). By definition, a weakly selective free ultrafilter is rapid.

Lemma 7.4. There exists a rapid maximal a-filter, if we assume the continuum hypothesis.

Proof. Let $\mathcal{U}$ be a rapid free ultrafilter on $\mathbb{N}$. Let

$$
\mathcal{F}:=\{S \in \mathcal{A}:\{n \in \mathbb{N}: 1 / n \in S\} \in \mathcal{U}\} .
$$

From the fact that $\mathcal{U}$ is a filter, it is straightforward to check that $\mathcal{F}$ is an a-filter. From the fact that $\mathcal{U}$ is rapid, resp. maximal, it is straightforward to check that $\mathcal{F}$ is a rapid, resp. prime a-filter. By theorem 6.12, it suffices to show that $\mathcal{F}$ closed. Let $S \in \overline{\mathcal{F}}$. As $S$ is a closed set, there exists a closed $T \succ S$ such that $\{n \in \mathbb{N}$ : $1 / n \in T\}=\{n \in \mathbb{N}: 1 / n \in S\}$. Since $T \in \mathcal{F},\{n \in \mathbb{N}: 1 / n \in T\} \in \mathcal{U}$. Hence also $S \in \mathcal{F}$.

Proposition 7.5. There exists a prime ideal in $\widetilde{\mathbb{K}}_{\mathrm{cnt}}$ which is both minimal and maximal, if we assume the continuum hypothesis.

Proof. Let $\mathcal{F}$ be a rapid maximal a-filter. By theorem $7.2, I(\mathcal{F})$ is closed, hence $I(\mathcal{F})$ is both a minimal and maximal prime ideal by corollary 6.14 .

\section{$8 z$-ideals}

As the notion of $z$-ideal in the $\operatorname{ring} \mathcal{C}(X)$ of continuous functions on a topological space $X$ can be expressed by a purely algebraic condition [7, 4A], G. Mason [9] used this condition to define a $z$-ideal of any commutative ring $R$ with 1 . 
Definition 8.1. Denoting by $\mathcal{M}(a)=\{M$ max. ideals of $R: a \in M\}, I \unlhd R$ is $a$ $z$-ideal if

$$
(\forall a \in R)(\forall b \in I)(\mathcal{M}(a)=\mathcal{M}(b) \Rightarrow a \in I)
$$

We proceed to show a similar characterization as for $z$-ideals in $\widetilde{\mathbb{K}}$. As in [13], we denote $Z(a):=\left\{S \in \mathcal{S}: a_{\mid S}=0\right\}$.

Theorem 8.2. Let $a, b \in \widetilde{\mathbb{K}}_{\mathrm{cnt}}$. Then $\mathcal{M}(a) \subseteq \mathcal{M}(b) \Longleftrightarrow Z(a) \subseteq Z(b)$.

Proof. $\Rightarrow$ : let $S \in Z(a) \backslash Z(b)$, i.e., $a_{\mid S}=0$ and $b_{\mid S} \neq 0$. By lemma 3.4, there exists $T \in \mathcal{S}$ with $T \subseteq S$ such that $b_{\mid T}$ is invertible. Let $M$ be a maximal ideal containing $I:=\left\{x \in \widetilde{\mathbb{K}}_{\text {cnt }}: x_{\mid T}=0\right\} \triangleleft \widetilde{\mathbb{K}}_{\text {cnt }}$. Since $a_{\mid S}=0$, also $a_{\mid T}=0$, hence $a \in M$. Suppose that $b \in M$. Since $b_{\mid T}$ is invertible, $b_{\mid U}$ is invertible for some $U \succ T$. By Urysohn's lemma, there exists $x \in \widetilde{\mathbb{K}}_{\mathrm{cnt}}$ such that $x_{\mid T}=0$ and $x_{\mid U^{c}}=1$. Hence $x \in I \subseteq M$, and $\bar{x} x+\bar{b} b=|x|^{2}+|b|^{2} \in M$ would be invertible, a contradiction. We conclude that $M \in \mathcal{M}(a) \backslash \mathcal{M}(b)$.

$\Leftarrow$ : let $M \in \mathcal{M}(a) \backslash \mathcal{M}(b)$, so $a \in M$ and $b \notin M$. As $M$ is maximal, $M+b \widetilde{\mathbb{K}}_{\mathrm{cnt}}=\widetilde{\mathbb{K}}_{\mathrm{cnt}}$. Let $m \in M$ and $c \in \widetilde{\mathbb{K}}_{\mathrm{cnt}}$ such that $m+b c=1$. As $b c, m \in \widetilde{\mathbb{K}}$, there exists $S \subseteq(0,1]$ such that $(b c)_{\mid S}$ and $m_{\mid S^{c}}$ are invertible [13, Lemma 4.1]. Hence also $b_{\mid S}$ is invertible. Suppose that $a_{\mid S}$ is invertible. Then $\bar{a} a+\bar{m} m=|a|^{2}+|m|^{2} \in M$ would be invertible, a contradiction. By lemma 3.4, there exists $T \in \mathcal{S}$ with $T \subseteq S$ such that $a_{\mid T}=0$. We conclude that $T \in Z(a) \backslash Z(b)$.

Corollary 8.3. $I \unlhd \widetilde{\mathbb{K}}_{\mathrm{cnt}}$ is a z-ideal iff

$$
\left(\forall a \in \widetilde{\mathbb{K}}_{\mathrm{cnt}}\right)(\forall b \in I)(Z(a)=Z(b) \Rightarrow a \in I) .
$$

\section{Proposition 8.4.}

1. For $I \unlhd \widetilde{\mathbb{K}}_{\mathrm{cnt}}$,

$$
\begin{aligned}
I_{z}: & =\left\{x \in \widetilde{\mathbb{K}}_{\mathrm{cnt}}:(\exists a \in I)(Z(x)=Z(a))\right\}=\left\{x \in \widetilde{\mathbb{K}}_{\mathrm{cnt}}:(\exists a \in I)(Z(x) \supseteq Z(a))\right\} \\
& =\left\{x \in \widetilde{\mathbb{K}}_{\mathrm{cnt}}:(\exists a \in I)(\mathcal{M}(x)=\mathcal{M}(a))\right\}=\left\{x \in \widetilde{\mathbb{K}}_{\mathrm{cnt}}:(\exists a \in I)(\mathcal{M}(x) \supseteq \mathcal{M}(a))\right\}
\end{aligned}
$$

is the smallest $z$-ideal containing $I$. We call it the z-closure of $I$. I is a z-ideal iff $I=I_{z}$.

2. For $I \unlhd \widetilde{\mathbb{K}}_{\mathrm{cnt}}, I \subseteq \sqrt{I} \subseteq I_{z}$. Hence $(\sqrt{I})_{z}=I_{z}$ and every $z$-ideal is radical. $A$ (proper) $z$-ideal is prime iff it is pseudoprime.

Proof. As in [13, Prop. 4.3].

Proposition 8.5. Every closed ideal $I \triangleleft \widetilde{\mathbb{K}}_{\mathrm{cnt}}$ is a z-ideal.

Proof. $I$ is an intersection of maximal ideals (proposition 6.15), hence a $z$-ideal [9].

\section{Proposition 8.6.}

1. For a family $\left(I_{\lambda}\right)_{\lambda \in \Lambda}$ of ideals $I_{\lambda} \unlhd \widetilde{\mathbb{K}}_{\mathrm{cnt}},\left(\sum_{\lambda \in \Lambda} I_{\lambda}\right)_{z}=\sum_{\lambda \in \Lambda}\left(I_{\lambda}\right)_{z}$. In particular, the sum of a family of $z$-ideals is a z-ideal. 
2. For $I, J \unlhd \widetilde{\mathbb{K}}_{\mathrm{cnt}}, I_{z} \cap J_{z}=(I \cap J)_{z}$.

3. For $I \unlhd \widetilde{\mathbb{K}}_{\mathrm{cnt}}, I^{z}:=\left\{x \in \widetilde{\mathbb{K}}_{\mathrm{cnt}}:\left(x \widetilde{\mathbb{K}}_{\mathrm{cnt}}\right)_{z} \subseteq I\right\}$ is the largest $z$-ideal contained in I. We call it the z-part of $I$. I is a z-ideal iff $I=I^{z}$.

4. For a family $\left(I_{\lambda}\right)_{\lambda \in \Lambda}$ of ideals $I_{\lambda} \unlhd \widetilde{\mathbb{K}}_{\mathrm{cnt}}, \bigcap_{\lambda \in \Lambda} I_{\lambda}^{z}=\left(\bigcap_{\lambda \in \Lambda} I_{\lambda}\right)^{z}$. In particular, the intersection of a family of $z$-ideals is a $z$-ideal.

5. For $I \unlhd \widetilde{\mathbb{K}}_{\mathrm{cnt}}, m(I) \subseteq I^{z} \subseteq I^{\sqrt{ }} \subseteq I$. In particular, every pure ideal of $\widetilde{\mathbb{K}}_{\mathrm{cnt}}$ is a $z$-ideal. If $I \triangleleft \widetilde{\mathbb{K}}_{\mathrm{cnt}}$ is pseudoprime, then $I^{z}$ is prime.

Proof. 1. First, we show that $(I+J)_{z}=I_{z}+J_{z}$.

Let $x \in(I+J)_{z}$. Hence there exist $a \in I, b \in J$ such that $Z(x)=Z(a+b)$. Let $\left(\alpha_{\varepsilon}\right)_{\varepsilon}$, resp. $\left(\beta_{\varepsilon}\right)_{\varepsilon}$, be representatives of $|a|$, resp. $|b|$, with $\alpha_{\varepsilon} \neq 0$ and $\beta_{\varepsilon} \neq 0$ for all $\varepsilon$. Let $S:=\left\{\varepsilon \in(0,1]: \alpha_{\varepsilon}<2 \beta_{\varepsilon}\right\}$ and $T:=\left\{\varepsilon \in(0,1]: \beta_{\varepsilon}<2 \alpha_{\varepsilon}\right\}$. As $\alpha_{\varepsilon} \neq 0$ and $\beta_{\varepsilon} \neq 0$, $S \cup T=(0,1]$. By lemma 6.3, there exist $V \prec S, U \prec T$ such that $U \cup V=(0,1]$. By Urysohn's lemma, there exists $y, z \in \widetilde{\mathbb{R}}_{\text {cnt }}$ such that $y_{\mid V}=1, y_{\mid S^{c}}=0, z_{\mid U}=1$ and $z_{\mid T^{c}}=0$ and $0 \leq y, z \leq 1$. Then $y+z \geq 1$. Hence there exists $u \in \widetilde{\mathbb{R}}_{\text {cnt }}$ such that $(y+z) u=1$.

Now let $W \in Z(a)$, i.e., $a_{\mid W}=0$. As $|b|_{\mid T} \leq 2|a|_{\mid T}$, also $b_{\mid T \cap W}=0$. Hence $T \cap W \in$ $Z(a+b)=Z(x)$, i.e. $x_{\mid T \cap W}=0$. Hence $x z u_{\mid W}=x z u_{\mid(W \cap T) \cup(W \backslash T)}=0$. Thus $Z(a) \subseteq$ $Z(x z u)$. As $a \in I, x z u \in I_{z}$. Similarly, $x y u \in J_{z}$. Hence $x=x y u+x z u \in I_{z}+J_{z}$.

For arbitrary sums, the result follows as in [13, Prop. 4.4].

2-4. As in [13, Prop. 4.4].

5. We show that $m(I) \subseteq I^{z}$. Let $x \in m(I)=I(\mathcal{F}(I))$. Then there exists $S \in \mathcal{F}(I)$ such that $x_{\mid S}=0$. Let $y \in\left(x \widetilde{\mathbb{K}}_{\text {cnt }}\right)_{z}$. Then also $y_{\mid S}=0$, so $y \in I(\mathcal{F}(I)) \subseteq I$. Thus $\left(x \widetilde{\mathbb{K}}_{\mathrm{cnt}}\right)_{z} \subseteq I$. The other statements follow as in [13, Prop. 4.4] (using [5, Prop. 4.29]).

Remark 8.7. There are $z$-ideals that are not closed (e.g., consider a minimal prime ideal that is not maximal).

It is well known that $\widetilde{\mathbb{K}}$ is complete for the sharp topology [12]. Similarly, we have:

Theorem 8.8. $\widetilde{\mathbb{K}}_{\mathrm{cnt}}$ is complete for the sharp topology.

Proof. Since $\widetilde{\mathbb{K}}_{\text {cnt }} \subseteq \widetilde{\mathbb{K}}$ and $\widetilde{\mathbb{K}}$ is complete, we show that $\widetilde{\mathbb{K}}_{\text {cnt }}$ is closed in $\widetilde{\mathbb{K}}$. Let $x_{n} \in \widetilde{\mathbb{K}}_{\text {cnt }}$ with continuous representative $\left(x_{n, \varepsilon}\right)_{\varepsilon}$ such that $x_{n} \rightarrow x \in \widetilde{\mathbb{K}}$. By taking a subsequence, we may assume that for each $n \in \mathbb{N}$,

$$
\left|x_{n, \varepsilon}-x_{\varepsilon}\right| \leq \varepsilon^{n}, \quad \forall \varepsilon \leq \varepsilon_{n} .
$$

W.l.o.g., $\left(\varepsilon_{n}\right)_{n}$ is strictly decreasing and tends to 0 . Then let $u_{1, \varepsilon}:=x_{1, \varepsilon}$ and

$$
u_{n, \varepsilon}:= \begin{cases}x_{n, \varepsilon}-x_{n-1, \varepsilon} & \varepsilon \leq \varepsilon_{n+1} \\ 0, & \varepsilon>\varepsilon_{n}\end{cases}
$$

in such a way that $u_{n, \varepsilon}$ is continuous in $\varepsilon$ and $\left|u_{n, \varepsilon}\right| \leq\left|x_{n, \varepsilon}-x_{n-1, \varepsilon}\right|$ for each $\varepsilon \in(0,1]$. Then $s_{\varepsilon}:=\sum_{n=1}^{\infty} u_{n, \varepsilon}$ is a locally finite sum. Hence $\left(s_{\varepsilon}\right)_{\varepsilon}$ is continuous and for each 
$\varepsilon \in\left(\varepsilon_{n+1}, \varepsilon_{n}\right]$

$\left|s_{\varepsilon}-x_{\varepsilon}\right|=\left|\sum_{k=1}^{n} u_{k, \varepsilon}-x_{\varepsilon}\right| \leq\left|u_{n, \varepsilon}\right|+\left|x_{n-1, \varepsilon}-x_{\varepsilon}\right| \leq\left|x_{n, \varepsilon}-x_{\varepsilon}\right|+2\left|x_{n-1, \varepsilon}-x_{\varepsilon}\right| \leq 3 \varepsilon^{n-1}$.

Hence $x=\left[s_{\varepsilon}\right] \in \widetilde{\mathbb{K}}_{\mathrm{cnt}}$.

Theorem 8.9. Let $I \unlhd \widetilde{\mathbb{K}}_{\mathrm{cnt}}$ be a finitely generated ideal.

1. If I is radical (in particular, if $I$ is closed, pure or a $z$-ideal), then $I \in\left\{0, \widetilde{\mathbb{K}}_{\mathrm{cnt}}\right\}$.

2. $I_{z}=\bar{I}$

3. $m(I)=I^{z}$.

Proof. By [5, Lemma 4.5], $I$ is principal, i.e. $I=a \widetilde{\mathbb{K}}_{\mathrm{cnt}}$ for some $a \in \widetilde{\mathbb{K}}_{\mathrm{cnt}}$.

1. By [5, Prop. 4.28], $I$ is idempotent. Hence $a=a^{2} b$ for some $b \in \widetilde{\mathbb{K}}_{\text {cnt }}$. Thus $a b$ is idempotent. So either $a b=0$, whence $a=a^{2} b=0$ and $I=0$, or $a b=1$, whence $I=\widetilde{\mathbb{K}}_{\text {cnt }}$.

2. Let $x \in \bar{I}$, i.e. $x=\lim _{n \rightarrow \infty} x_{n}$ for some $x_{n} \in I$. Let $S \in Z(a)$, i.e. $a_{\mid S}=0$. Then also $x_{n \mid S}=0$ for each $n \in \mathbb{N}$, hence also $x_{\mid S}=0$, i.e. $S \in Z(x)$. Thus $x \in I_{z}$. The converse inclusion holds by proposition 8.5.

3. Let $x \in \widetilde{\mathbb{K}}_{\text {cnt }} \backslash m(I)=I(\mathcal{F}(I))$. Then for each $S \in \mathcal{F}(I), x_{\mid S} \neq 0$. In particular, let $\left(a_{\varepsilon}\right)_{\varepsilon}$ be a (continuous) representative of $a$ and $L_{n}:=\left\{\varepsilon \in(0,1]:\left|a_{\varepsilon}\right|>\varepsilon^{n}\right\}$. Then $L_{n}^{c} \in \mathcal{F}(I)$, so $x_{\mid L_{n}^{c}} \neq 0$. By lemma 3.4, there exist $T_{n} \in \mathcal{S}$ with $T_{n} \subseteq L_{n+1}^{c}$ and $x_{\mid T_{n}}$ is invertible. By lemma 3.5, there exist $S_{n} \succ T_{n}$ such that $x_{\mid S_{n}}$ is invertible (as $T_{n} \prec L_{n}^{c}$, we may assume $S_{n} \prec L_{n}^{c}$ ). By Urysohn's lemma, there exist $y_{n} \in \widetilde{\mathbb{K}}_{\text {cnt }}$

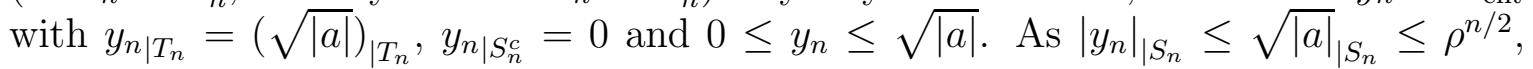
$y:=\sum_{n=1}^{\infty} y_{n} \in \widetilde{\mathbb{K}}_{\text {cnt }}$ exists $\left(\widetilde{\mathbb{K}}_{\text {cnt }}\right.$ is a complete ultrametric space $)$. We show that $y \in\left(x \widetilde{\mathbb{K}}_{\mathrm{cnt}}\right)_{z}$.

Let $U \in Z(x)$, i.e., $x_{\mid U}=0$. Then $0 \notin \overline{U \cap S_{n}}$, since $x_{\mid S_{n}}$ is invertible. Hence $y_{n \mid U}=0$. Then also $y_{\mid U}=0$, i.e., $U \in Z(y)$.

Also $y \notin I:|y|_{\mid T_{n}} \geq\left|y_{n}\right|_{\mid T_{n}}=\sqrt{|a|}_{\mid T_{n}} \geq\left(\rho^{-n / 2}|a|\right)_{\mid T_{n}}$ for each $n \in \mathbb{N}$. Hence $|y| \not \leq$ $\rho^{-N}|a|$ for any $N \in \mathbb{N}$, and thus $y \notin I$. Hence $\left(x \widetilde{\mathbb{K}}_{\mathrm{cnt}}\right)_{z} \nsubseteq I$, i.e., $x \notin I^{z}$.

Let $I \triangleleft \widetilde{\mathbb{K}}_{\mathrm{cnt}}$. Let $I^{\perp}=\left\{x \in \widetilde{\mathbb{K}}_{\mathrm{cnt}}: x y=0, \forall y \in I\right\}$. As in $\widetilde{\mathbb{K}}$, we have:

Proposition 8.10. Let $I \triangleleft \widetilde{\mathbb{K}}_{\mathrm{cnt}}$. Then

1. $I^{\perp}$ is closed.

2. $\bar{I} \subseteq I^{\perp \perp}$.

3. $\bar{I} \cap I^{\perp}=\{0\}$.

4. If $I$ is pseudoprime, then $I^{\perp}=\{0\}$. In particular, $\bar{I} \varsubsetneqq I^{\perp \perp}=\widetilde{\mathbb{K}}_{\mathrm{cnt}}$. 
Proof. 1. Let $x=\lim _{n \rightarrow \infty} x_{n}$, with $x_{n} \in I^{\perp}$. Then $x_{n} y=0, \forall n \in \mathbb{N}$, hence also $x y=0, \forall y \in I$. Thus $x \in I^{\perp}$.

2. If $x \in I$, then $x y=0, \forall y \in I^{\perp}$, so $I \subseteq I^{\perp \perp}$. By part 1 , also $\bar{I} \subseteq I^{\perp \perp}$.

3. If $x \in I \cap I^{\perp}$, then $x^{2}=0$, hence $x=0$. Hence also $I^{\perp} \cap \bar{I} \subseteq I^{\perp} \cap I^{\perp \perp}=\{0\}$.

4. Let $x \in I^{\perp}$. If $x \neq 0$, then there exists $T \in \mathcal{S}$ such that $x_{\mid T}$ is invertible. By lemma 3.5, there exists $S \succ T$ such that $x_{\mid S}$ is invertible. W.l.o.g. $S$ is closed, $T$ is open and $S^{c} \in \mathcal{S}$. As $\left(T^{c}\right)^{\circ} \cup S^{\circ}=(0,1]$ and $\mathcal{F}(I)$ is pseudoprime, either $T^{c} \in \mathcal{F}(I)$ or $S \in \mathcal{F}(I)$. In the first case, there exists $y \in I$ such that $y_{\mid T}=1$. As $x \in I^{\perp}, x y=0$, contradicting the fact that $(x y)_{\mid T}$ is invertible. In the second case, there exists $y \in I$ such that $y_{\mid S^{c}}=1$. Hence $x y=0$, and thus $x_{\mid S^{c}}=0$. As $(x z)_{\mid S}=1$ for some $z \in \widetilde{\mathbb{K}}_{\mathrm{cnt}}$, and $(x z)_{\mid S^{c}}=0, x z \in \widetilde{\mathbb{K}}_{\text {cnt }}$ is idempotent, and hence $x z=0$ (contradicting $(x z)_{\mid S}=1$ ) or $x z=1$ (contradicting $(x z)_{\mid S^{c}}=0$ ). Thus $x=0$.

Lemma 8.11. There exists $J \triangleleft \widetilde{\mathbb{K}}_{\text {cnt }}$ such that $J \neq\{0\}$ and $J^{\perp} \neq\{0\}$.

Proof. Let $S:=\bigcup_{n \in \mathbb{N}}\left(a_{n}, b_{n}\right)$ with $1>b_{1}>a_{1}>b_{2}>a_{2}>\ldots$ and $a_{n} \rightarrow 0$. Then there exists $x \in \widetilde{\mathbb{K}}_{\text {cnt }} \backslash\{0\}$ such that $x_{\mid S}=0$ and there exists $y \in \widetilde{\mathbb{K}}_{\text {cnt }} \backslash\{0\}$ such that $y_{\mid S^{c}}=0$. Let $J=\left\{x \in \widetilde{\mathbb{K}}_{\mathrm{cnt}}: x_{\mid S}=0\right\}$. Then $x \in J$ and $y \in J^{\perp}$.

Also as in $\widetilde{\mathbb{K}}$, the Hahn-Banach extension property does not hold in the following sense:

Theorem 8.12. Let $J \triangleleft \widetilde{\mathbb{K}}_{\text {cnt }}$ with $J \neq\{0\}$ and $J^{\perp} \neq\{0\}$. Let $I:=J+J^{\perp}$. Then there exists a continuous $\widetilde{\mathbb{K}}_{\mathrm{cnt}}$-linear map $\phi: I \rightarrow \widetilde{\mathbb{K}}_{\mathrm{cnt}}$ that cannot be extended to a $\widetilde{\mathbb{K}}_{\mathrm{cnt}}$-linear map $\psi: \widetilde{\mathbb{K}}_{\mathrm{cnt}} \rightarrow \widetilde{\mathbb{K}}_{\mathrm{cnt}}$.

Proof. Let $\phi(x+y):=x$, for each $x \in J$ and $y \in J^{\perp}$. As $J \cap J^{\perp}=\{0\}, \phi$ is defined unambiguously and is $\widetilde{\mathbb{K}}_{\mathrm{cnt}}$-linear. Further, $|\phi(x+y)|^{2}=|x|^{2} \leq|x|^{2}+|y|^{2}=$ $(x+y)(\bar{x}+\bar{y})=|x+y|^{2}$, for each $x \in J$ and $y \in J^{\perp}$. Hence $\phi$ is also continuous.

Now suppose that $\psi: \widetilde{\mathbb{K}}_{\mathrm{cnt}} \rightarrow \widetilde{\mathbb{K}}_{\mathrm{cnt}}$ is a $\widetilde{\mathbb{K}}_{\mathrm{cnt}}$-linear extension of $\phi$. Then for any $x \in J$, $x \psi(1)=\psi(x)=\phi(x)=x$. Hence $x(\psi(1)-1)=0$. Thus $\psi(1)-1 \in J^{\perp}$. Hence $\psi(1) \psi(1)-\psi(1)=\psi(\psi(1)-1)=\phi(\psi(1)-1)=0$. It follows that $\psi(1) \in \widetilde{\mathbb{K}}_{\mathrm{cnt}}$ is idempotent, hence $\psi(1)=0$ or $\psi(1)=1$. If $\psi(1)=0$, then $\psi=0$, and thus also $\phi=0$, whence $J=\{0\}$. If $\psi(1)=1$, then $\psi(x)=x$ for each $x \in \widetilde{\mathbb{K}}_{\text {cnt }}$, and thus also $\phi(y)=y$ for each $y \in J^{\perp}$, whence $J^{\perp}=\{0\}$.

Corollary 8.13. If $I \triangleleft \widetilde{\mathbb{K}}_{\text {cnt }}$ with $I \neq\{0\}$ and $I^{\perp} \neq\{0\}$, then $I+I^{\perp} \neq \widetilde{\mathbb{K}}_{\text {cnt }}$.

\section{References}

[1] J. Aragona, S. Juriaans. Some structural properties of the topological ring of Colombeau's generalized numbers. Comm. Algebra 29(5): 2201-2230 (2001).

[2] A. Bigard, K. Keimel, S. Wolfenstein. Groupes et anneaux réticulés. Lecture Notes in Math. vol. 608. Springer, 1977.

[3] D. Booth. Ultrafilters on a countable set. Ann. Math. Logic 2: 1-24 (1970). 
[4] F. Borceux, G. Van den Bossche. Algebra in a localic topos with applications to ring theory. Lecture Notes in Math. vol. 1038. Springer, 1980.

[5] A. Burtscher, M. Kunzinger. Algebras of generalized functions with smooth parameter dependence. Proc. Edinburgh Math. Soc. 55(1): 105-124 (2012).

[6] G. Choquet. Deux classes remarquables d'ultrafiltres sur N . Bull. Sci. Math. 92(2): 143-153 (1968).

[7] L. Gillman, M. Jerison. Rings of continuous functions. Springer Graduate Texts in Math. 43, 1960.

[8] L. Gillman, C.W. Kohls. Convex and pseudoprime ideals in rings of continuous functions. Math. Z. 72: 399-409 (1960).

[9] G. Mason. z-ideals and prime ideals. J. Algebra 26: 280-297 (1973).

[10] H. Matsumura. Commutative ring theory. Cambridge University Press (English translation), 1986.

[11] W. Rudin. Homogeneity problems in the theory of Cech compactifications. Duke Math. J. 23: 409-419 (1956).

[12] D. Scarpalézos. Colombeau's generalized functions: topological structures; microlocal properties. A simplified point of view. Université Paris 7 (1993).

[13] H. Vernaeve. Ideals in the ring of Colombeau generalized numbers. Comm. Alg. 38(6): 2199-2228 (2010). 